\title{
ELECTRON TRAJECTORIES IN PULSED RAOIATION FIELDS
}

Ted Einwohner and 8. A. Lippmann

UCID- -21055

DE87 009983

\section{Introduction}

The work reported here analyzes the dynamical behavior of an electron, initially at rest, when subjected to a radiation pulse of arbitrary, but integrable, shape. Thts is dor. by a general integration procedure that has been programmed in VAXIMA. Upon choosing a specific shape for the pulse, VAXIMA finds both the space-time trajectory and the four-momentum of the electron. These are ot ained in analytic or numerical form - or both - at the choice of the ser. Several examples of analytical and numerical solutions, for different pulse shapes, are given in the next Section.

As will be seen in the equations of motion (Eq. (1) below), the radiation pulses are functions of $t-z / c$, while the differentiations are with respect to $\tau$, the electron's proper time. Before VAXIMA can set to work, the relation between $t-2 / c$ and must be established, This rolation is especially stmple if the electron is at rest at some time; namely, the retarded time, $t-2 / c$, and the electron proper time, $\tau$, are both the same.

To show this, we note that

$$
\begin{aligned}
\frac{d}{d \tau} & =r \frac{d}{d t}, \\
r & =\frac{d \tau}{d t},
\end{aligned}
$$


and the relativistic equations of motion are

$$
\begin{aligned}
& \frac{d^{2} \underline{r}}{d \tau^{2}}=\frac{e}{m}\left(\gamma \underline{E}+\frac{1}{c} \frac{d \underline{r}}{d \tau} \times \underline{B}\right) f(t-z / c) \\
& \frac{d^{2} t}{d \tau^{2}}=\frac{d \gamma}{d \tau}=\frac{e}{m} \frac{d r}{d \tau} \cdot \underline{E} f(t-z / c) .
\end{aligned}
$$

For definiteness, we have taken the E-field to be (unit vectors indicated by a circumflex)

$$
\begin{aligned}
E(\underline{r}, t) & =\underline{E} f(t-z / c) \\
& =\ddot{x} E_{0} f(t-z / c) .
\end{aligned}
$$

The $\mathrm{B}-\mathrm{fie}$ id is then given by

$$
\begin{aligned}
\underline{B}(\underline{r}, t) & =\underline{B} f(t-z / c)=\underline{\tilde{z}} \times \underline{E}(\underline{r}, t) \\
& =\hat{y} E_{0} f(t-z / c) .
\end{aligned}
$$

The $z$ and $t$ equations in (1) imply that

$$
\frac{d^{2}}{d \tau^{2}}(t-z / c)=\frac{d}{d \tau}\left(r-\frac{1}{c} \frac{d z}{d \tau}\right)=0 .
$$

If the electron is at rest at any point in its trajectory, $Y=1$ at this point and the above integrates to

$$
\frac{d}{d \tau}(t-z / c)=\gamma-\frac{1}{c} \frac{d z}{d \tau}=1
$$

from which, we see that

$$
t-z / c=\tau
$$

In the last equation, the inessential additive constant has been set equal to zero.

$$
\text { Nuth }
$$


The relativistic equations of motion now become

$$
\begin{aligned}
& \frac{d^{2} \underline{r}}{d \tau^{2}}=\frac{e}{m}\left(r \underline{E}+\frac{1}{c} \frac{d \underline{r}}{d \tau} \times \underline{B}\right) f(\tau) \\
& \frac{d^{2} t}{d \tau^{2}}=\frac{d Y}{d \tau}=\frac{e}{m c^{2}} \frac{d \underline{r}}{d \tau} \cdot \underline{E} f(\tau)
\end{aligned}
$$

These equations are in a form suitable for integration by MACSYMA, provided that the function $f(\tau)$ is integrable.

An important physical result follows immediately from Equation (2). Since $r-1 \geq 0$, we also have $\frac{d z}{d \tau} \geq 0$. Thus, the electron's velocity in the z-direction is always positive or zero. The electron never goes backward. The famous figure-8 electron motion does not occur in this case, but only if the mean electron motion is zero.

Another important physical result is obtained in the numerical studies in section 2. If the electron is in a field of limited extent in the $x$-direction, say the field from a parabolic reflector, for example, the ultimate $z$-momentum acquired by the elactron is then limited by the $x$-displacement; the $z$-momentum increases only while the $x$-displacement of the electron is less than the width of the beam in the $x$-direction.*

What about previous work? Here, our knowledge is somewhat meager. We have not made any attempt to explore the literature in detail, but we have looked up the granddaddy of electron-radiation processes - G. A. Schott's Adam Prize Essay, published in 1912 by Macmillan under the title

\footnotetext{
We are indebted to Ray kidder for this remark.
} 
"Electromagnetic Radiation" - with no positive results. However, there was a flurry of activity during the $1960^{\prime} s^{1-4}$ and also more recent work at LLNL. 5 These papers integrated the equations of motion to find the particle momenta; the final integration to yield the particle trajectories was not carried out.

\section{The Vaxima Program:}

The program can be round on the J-Vax as rthe/pub/relchgmoj. One can obtain a copy by doing the UNIX command $C P \sim$ the/pub/relchgmoj relchgmoj. He will then have his own copy. The ungainly last word, relchgmoj. can be replaced by any name one chooses. A copy also resides on the III-crg vax as $\sim$ the/pub/relchgmoj.

Usage:

There are three principal Vaxima functions available, allowing for greater flexibility in specifying the initial conditions and the form of the field: rel_chg_moj, rel_chg_moj_nz, and rel_chg_moj_3reg. In all functions, the electric field is assumed to be in the $x$ direction and propagating along the $z$ direction. Its form is $E_{0} f(t-z / c)$. The output forms for the motion of the particle will involve the parameter alf $=q E_{0} / m$, where $m$ is the mass of the charged particle and $q$ is its charge. The simplest function, rel_chg_moj(ff, tau), assumes that the particle is at rest at $z=0$ for $l a b$ and proper time $=0$. The argument tau is the name one wants to use for the proper time. The argument $f f$ is the 
name of a previously defined function. An example of a previously defined function is the built-in function, norm(t), which is defined as $\sin (w t)$. You can use it by using the word norm in place of $\mathrm{ff}$. Other built-in functions are

$$
\begin{aligned}
& \operatorname{dampsin}(t)=e^{-k t} \sin (\omega t), \quad[k>0] \\
& \operatorname{rek}(t)=(t / T) e^{-(t / T)} \\
& \operatorname{gauss}(t)=e^{-\left(t^{\star} t\right) /\left(2^{\star} s^{\star} s\right)}, \\
& \operatorname{zero}(t)=0 .
\end{aligned}
$$

You can define other field functions for yourself, following the template, e.g.:

$$
\text { anyname }(t):=x_{e}^{\star}(-t)+c \star x e(-2 \star t) \$
$$

Note the combined colon and equal sign is required for function definition. The combined symbol, \%e, denotes the base of natural logarithms. All lines defining functions should end with a followed by a carriage return. A built-in function or one you define can be used anywhere. Unfortunately, complicated functions involving gaussians do not go to correct limits as $t$ become infinite, a bug in the system at present. What this means is that expressions involving gaussians are not simplified and unmanageably long expressions result.

The second function, rel_chg_moj_nz ( $f m, f p, t a u, t i)$, is sightly more complicated and allows more general initial conditions and a more general form of the electric field. The particle velocity can be set to 
zero for any negative proper time, ti. The time can even be $-\infty$, by using the symbol minf for ti. The function was designed for possibly infinite time ranges and anticipates the possibility that the functional form of the electric field might change as its argunent changes from negative to positive. For example, the field might depend on the absolute value of its argument. Such forms cannot be handled simply. That is why rel_chg_moj_nz allows the specification, fm, for the field when its argument is negative and $f p$ when its argument is positive.

The third function, rel_chg_moj_3reg (fm, fi, fp, tau, ti, t1), allows three regions of independent definition of the fleld. The first is for negative proper time. The second is for proper time from zero to the time specified by the argument, tl. The third region is from $t$ i on. The functional forms of the field in these regions are given by the arguments $f m, f i$, and $f p$ respectively. As before, the argument, $t l$, is the negative time for which the particle velacity is zero, and the argument tau is the symbal used to Jenote proper time.

\section{Qutput}

For the simplest function, rel_chg_moj, the answers will appear in two forms. The first will be a display which is easy to read but cannot be mantpulated further; the second is a list which is harder to read but which can be further manipulated and in particular numerically evaluated. The display is of the form of a sequence of equalities of the form:

$$
\text { symbolname }=\text { expression }
$$


where symbolname is one of the symbols: $x, 2$, gam, or to denote lab $x$ coordinate, $z$ coordinate, gamma or lab time, and expression is its algebraic value, in terms of the input parameters and the proper time, tau.

The list, subject to further manipulation is of the form: $[x, z, g a m, t]$. For the more complicated function rel_chg_moj_nz each of the items: $x$, 2 , gam, $t$, is separated into three parts, corresponding to negative, zero. and positive proper time. Thus in the display $x$ is shown as three equations: $x m=, x 0=$, and $x p=$. In the output list, the entry corresponding to $x$ is replaced by a list of three items, corresponding to $x m, x 0$, and $x p$.

An auxiliary function, ev, generally avallable in Vaxima, allows partial or full numerical evaluation.

\section{An Interactive Vaxima Session to Calculate Electron Trajectories}

The following interactive session was actually walked through by two theoretical physicists, who use computers rarely and used Vaxima for the first time. It uses the simplest case of the aforeinentioned function and performs numerical evaluation.

User inputs are in plaintext, computer outputs are underlined. comments are indented. An expression in sbrackets> is the meaning of the expression, not the literal text. The symbol cr means carriage return. It will be assumed at the end of the input line after a few examples. 
j yli cr The $j$ means that, after proper connection with ocTopus, you want the J-Vax (Johnson's Vax). Your input $j$ will be echoed as idj. Then you type your login name for the j-Vax. Here the login name is yli.

yli The login name is echoed.

Password: swordfish $\mathrm{cr}$

You are asked for your password. Here swardfish is typed in as a password, but that is not echoed. (We don't repeat Marxian madness here.)

Last login: Mon Mar 17 14:45:42 on tty04

LLNL $\leq e t c \geq$

stime $>\langle$ how long up $><$ computer usage statistics $>$

scurrent activities of users

sdate $\rangle\langle$ time $>$

The last three sets of outputs are for the current setting of the user's UNIX . login file. You can change which UNIX functions are performed by editing the login file.

[]-rek<prompt-number $>]$ savaxima

This is a prompt from the UNIX system signifying that it is ready for a new task. The prompt number increases by one for each new task. This form of prompt is not automatic but must be edited into your . login file. Svaxima is the user input to the UNIX system to start up the raxima file. From now on the symbol or will be omitted. 
Vaxima 2.10

Fri March $30 \quad 17: 40: 51 \quad 1984$

Now we are in the Vaxima pragram and must follow its rules:

1. Prompts are of the form: the letter $c$ follows by an integer.

2. User input must with either: a follows by a $\mathrm{cr}$ or: a; follows by a cr. The former is interpreted as do the task but don't show me the result. The latter is interpreted as do the task and show me the output.

3. In the latter case the output is labeled by a the letter d followed the same integer that was in the prompt.

(c1) load (reichmoj) \$

The Vaxima instructions in the file relchmoj are now loaded in.

(c2) ttyoff:true $\$$

The first Vaxima instruction in the file relchmoj is to turn off display off nost of the subsequent instructions until the file is finished loading.

(cB) $\ldots \ldots$

(c1).....

Batching done

(c13)_rel_chg_moj (norm, tau);

We want to try a sinusoidar pulse, sin ( $w t)$, and we want to use the symbol tau for the proper time. 
He want to see the answer. Here the answer is a list of four algebraic expressions enclosed in square brackets. Before showing the answer, a display of four equations is shown:

$x=\ldots$

$z=\ldots$

gam $=\ldots$

$t=\ldots$

These are the $x$ coordinate, the $z$ coordinate, the relativistic gama and the lab frame time. These quantities are repeated in the answer for later manipulation.

$$
x=\frac{\operatorname{alf}(\tan w-\sin (\tan w))}{w^{2}}
$$

- $\langle$ etc

(d13)

$\left[\frac{\operatorname{a}] f(\tan w-\sin (\tan w))}{w^{2}}\right.$,

$\frac{\operatorname{alf}{ }^{2}(\sin (2 \operatorname{ta} u w)+4(\operatorname{ta} \omega-2 \sin (\tan w))+2 \operatorname{ta} u w}{8 c w^{2}}$.

$$
\begin{aligned}
& \frac{\operatorname{alf}^{2}(1-\cos (\tan w))^{2}}{2 c^{2} w^{2}}+1 \\
& \left.\frac{\operatorname{alf}\left(\sin (2 \tan w)^{3}+4(\operatorname{tau} w-2 \sin (\operatorname{ta} w)+2 \operatorname{ta} u w)+\operatorname{tau} u\right.}{a c^{2} w^{j}}\right]
\end{aligned}
$$

A1l formulas and equations here are part of the output and should be underlined. 
$(c] 4) e v(d 13, w: 1, b 9, \operatorname{tau}: 1, b-9, c: 3, b 10, a 1 f: 4.8 b-10 * v /(300 . * 9.11 b-28), b f$ loat $)$; Evaluate the expression labeled (d13) in the following environment:

1) The angular frequency, $w$, is set to $1 . \times 10^{9}$ in double precision.

2) The proper time, tau, is $1 . \times 10^{-9} \mathrm{sec}$.

3) The constant, aif, is writte., in terms of the electron mass, the electron charge, the potential in volts, and a conversion const. from volts to cgs, units.

4) bfloat means combine numbers in double precision.

Warning: Float to bigfloat conversion of 300.0

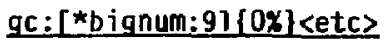

Symbolic calculations link words in memory. When intermediate calculations are finished, the linkages are no longer needed. When space runs out, it is renewed by purging the unneeded linkages. The gc message tells us of the purging.

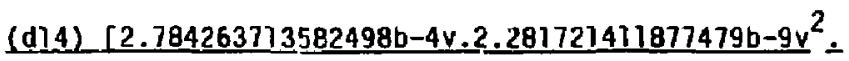

- $\quad 3.621390490947548 b-10 v^{2}+1.0 b 0$.

$7.605738039591598 b-20 v^{2}+1.0 b-91$

These are formulas in $v$, the voltage. Substituting numerical values for $v$ completes sthe numerical evaluation.

(ci5) quit()s We leave Vaxima.

[J-rek <next prompt number>l logout

We leave the J-machine.

[bye] 


\section{Further Examples of Usage:}

The following are annotated Vaxima sessions, giving examples of the three principal Vaxima functions: rel_chg_moj, rel_chg_moj_nz, and re1_chg_moj_3reg. Notes for the following pages are:

[1] Here the simplest function re1_chg_moj is invoked with the field an exponentially damped sin. The symbol for the proper time is taken to be tau.

[2] Vaxima realizes that it must do integrals of an exponential multiplied by a sin, which it interprets as the real part of integrals of complex exponentials. Accordingly, it calls the real part function from its library.

[3] The values for $2, x$, gamma, and the laboratory time are displayed. Note that they are sometimes not as simplified as they could be. This is because there is no absolute definition of simplest, and general purpose functions cannot anticipate which function would appear simplest in a given situation.

[4] Here begins the list of the functions displayed above. This list is capable of further manipulation, e.g. numericat evaluation. The display is not.

[5] This is the other example of the function rel_chg_moj applied to the field, rek, defined above. As before, the symbol for proper time is taken to be tau.

[6] Vaxima allows the input and output of a session to be stored in a textfile. Here the file is called baldemo, and the function closefile says to store no more of the interactive session there. 
[7] Here is an example of the more complicated function rel_chg_moj_nz, which allows the initial condition of zero velocity at some other proper time than zero. It also allows the user to use different functional forms for the field for positive and negative proper times. In this example the initial condition is for proper time minf, $-\infty$, See line c24 below. The field function for negative time is fim and for positive time $\mathrm{fp}$. These functions are defined by the user in lines c21 and c22.

[8] The functional form of integrals depends on the sign of parameters, or combinations of parameters. If these signs are unknown, Vaxima will ask. I told Vaxima that $k$ was to be positive.

[9] I had forgotten that the information of positive $k$ was already known. [jo] This is garbage collection information. (Vaxima and other symbolic manipulation systems calculate by linking memory cells.) When intermediate answers are obtained linked memory cells are freed for further use. This freeing is called garbage collection. The user is notified about garbage collection with this type of message. This message gives useful information about how well this problem is running and whether it will run out of space. However, it confuses the printing of answers. Garbage collection notification can be surpressed with the Vaxima command gcprint:false. That was done for the previous examples. [11] I had forgotten to specify the sign of $\mathrm{w}$, therefore I was asked. [12] $\mathrm{p} ;$ means positive (in like manner $\mathrm{n}_{\text {; }}$ or $z_{i}$ ). The full words, followed by a semicolon are also accepted.

[13] The last example is for the three region function, rel_chg_moj_3reg. There are to be three adjacent intervals for the preper time: the first from $\rightarrow$ to zero, the second from zero to the 
last argument of re1_chg_moj_3reg, in this example $\pi / W$, the third from $\pi / W$ to $+\infty$. The functional form for the field in the three consecutive intervals is given by the first three arguments of rel_chg_moj_3reg, in this example the previously defined fm and norm and a function fpp defined here. This form is chosen to make the field cont inuous at $\pi / W$.

[14] Vaxima allows several commands to be given in sequence by delimiting the commands with commas and plactng them in parentheses. The answer returned is the result of the last command.

Work performed under the auspices of the U.S. Uepartinent of Energy by the Lawrence Livermore National Laboratory under contract number W-7405-ENG-48. 


\section{REFERENCES}

1. J. J. Sanderson, Physics Letters 18, 114 (1965).

2. T. W. B. Kibble, Phys. Rev. 138B, 740 (1965).

3. Lowel1 S. Brown and T. W. B. Kibble, Phys. Rev. 133A, 705 (1964).

4. E. S. Sarachik and G. T. Schappert, Phys. Rev. 1D, 2738 (1970).

5. 3. Denavit and D. E. Nielsen, Jr.. Appendix 1 of COPD 84-38, February 21,1984 , unpub11shed. 


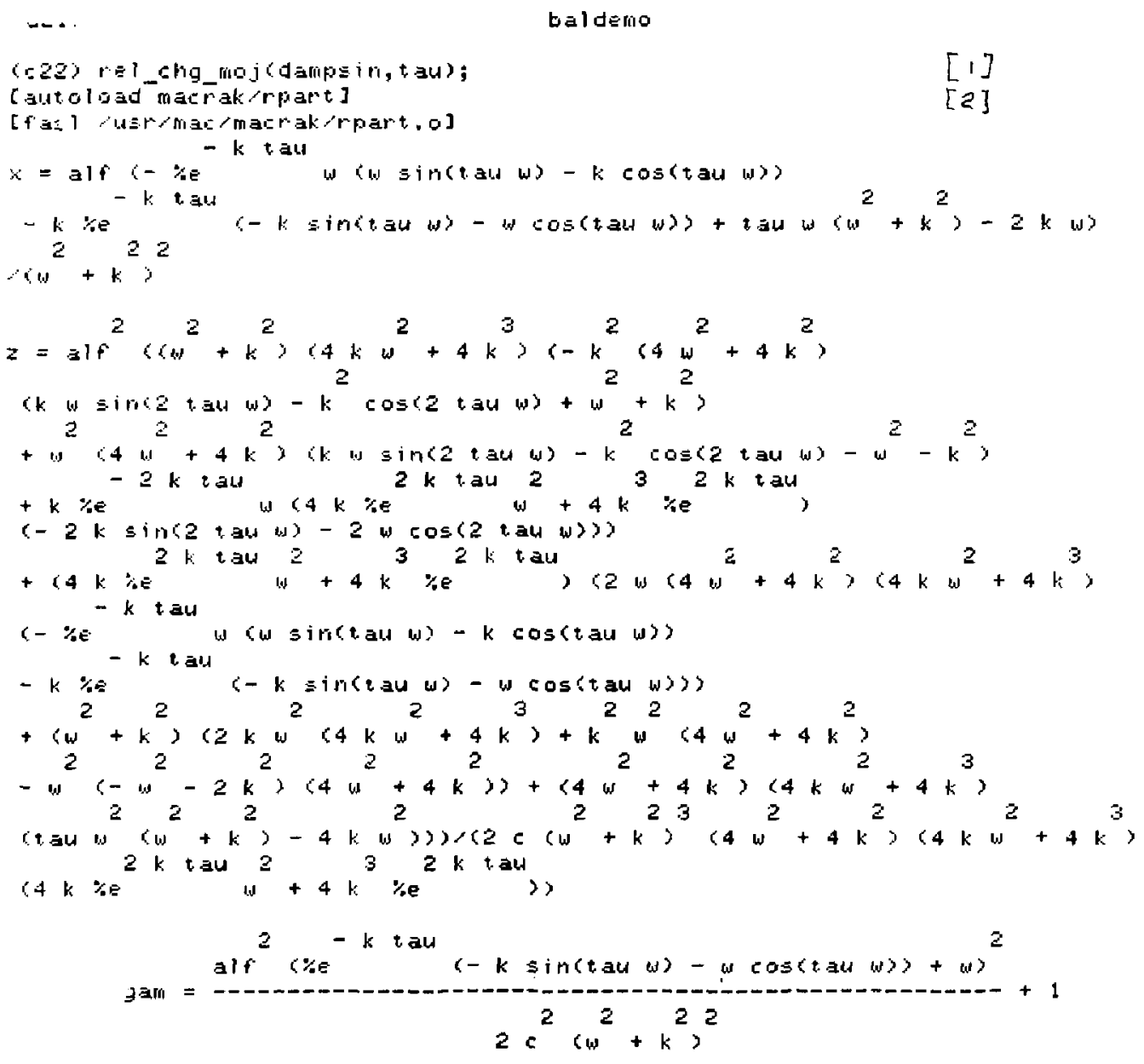




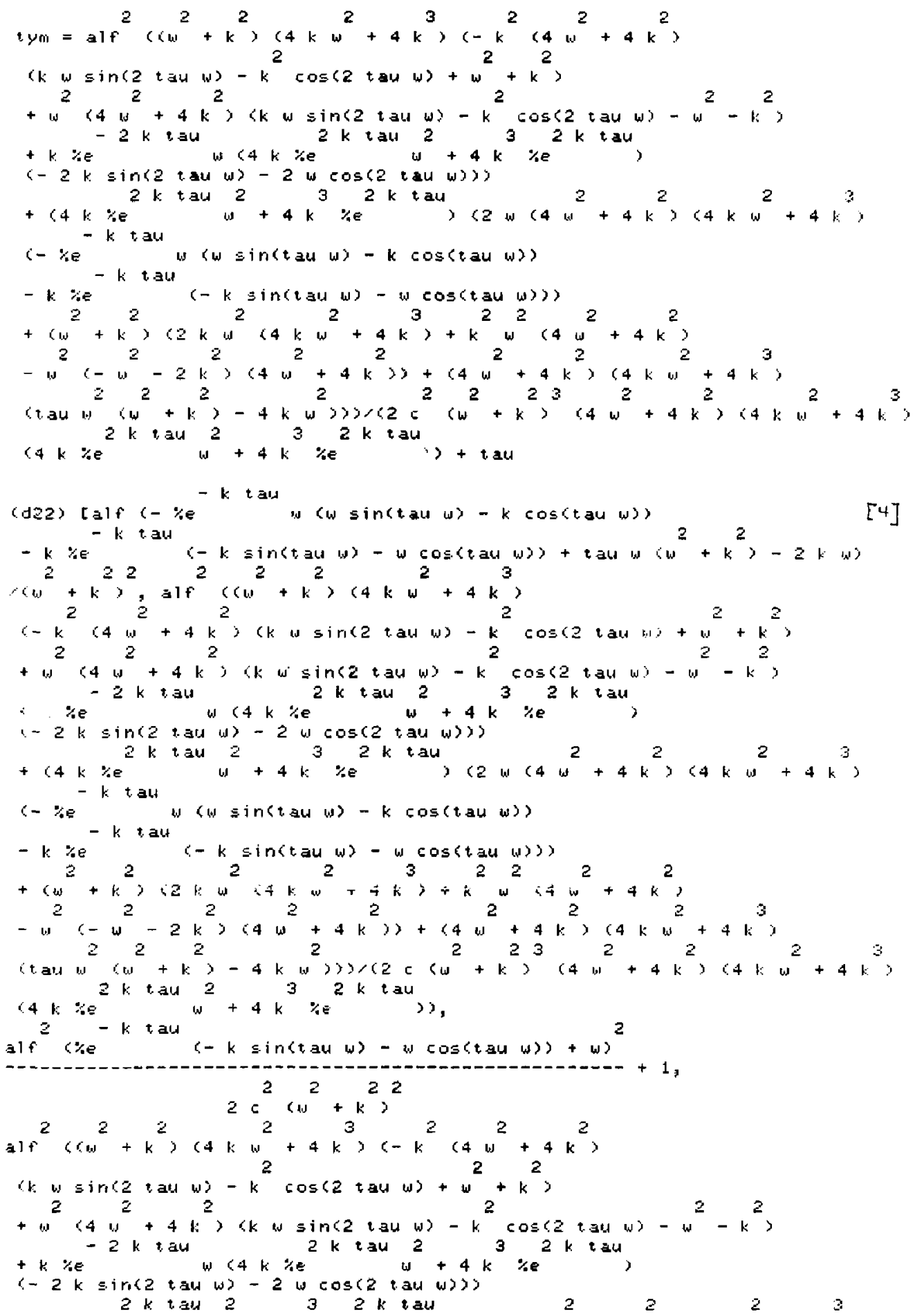




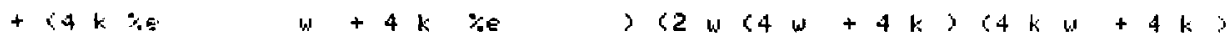

$\left(-x e^{-k t a u} w(\omega \sin (t a \omega \omega)-k \cos (t a \omega \omega)\right.$

$-k$ tau

$-k \quad(-k \equiv i n\langle t a w w)-w \cos (t \operatorname{ag} w)\rangle\rangle$

$+\left(w^{2}+k^{2}\right)\left(2 w^{2}\left(4 k^{2} w^{2}+4 k^{5}\right)+k^{2} w^{2}\left(4 w^{2}+4 k^{2}\right)\right.$

$-2\left(-w^{2}-2 k^{2}\right)\left(4 u^{2}+4 k^{2}\right)+\left(4 w^{2}+4 k^{2}\right)\left\langle 4 w^{2}+4 k^{3}\right.$

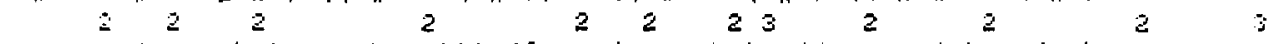

(t)

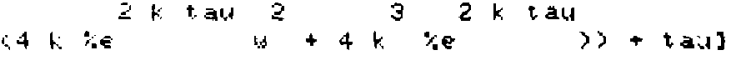

(c23) rel_chg_rojo (rek,tau);

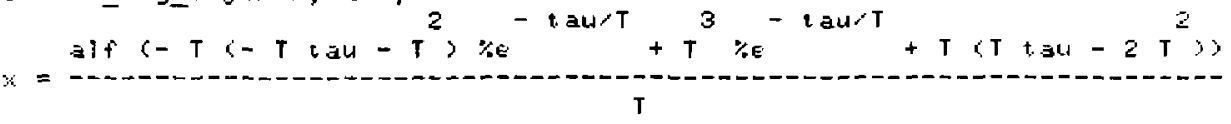

$z=31 f^{2} c T^{2}\left(T^{3} \% \theta^{-t a u T^{2}}-T\left(-T \operatorname{tau}-T^{2}\right) \% e^{-2 a u T^{T}}\right)$

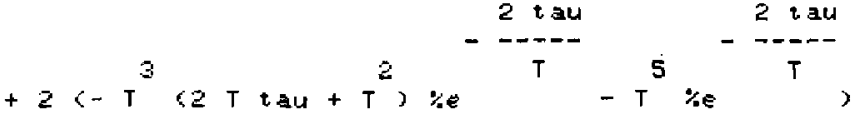

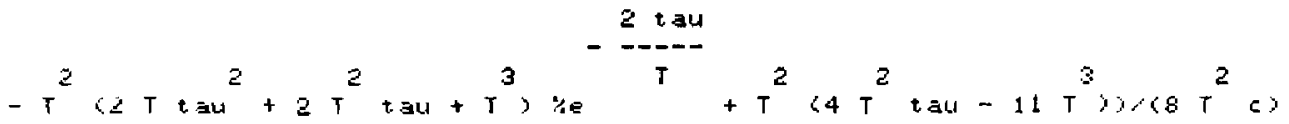

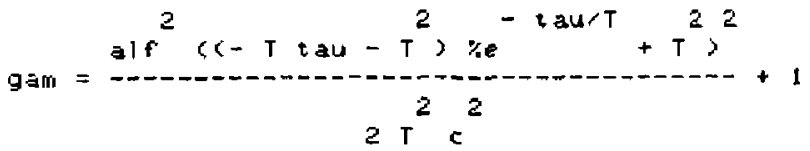

$$
\begin{aligned}
& t y m=a l f^{2}\left(g T^{2}\left(T^{3} z e^{-t a w} T-T\left(-T t a u-T^{2}\right) \Leftrightarrow e^{-t a u r T}\right)\right.
\end{aligned}
$$

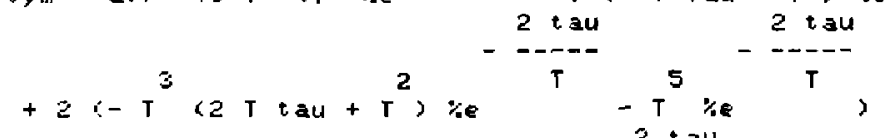

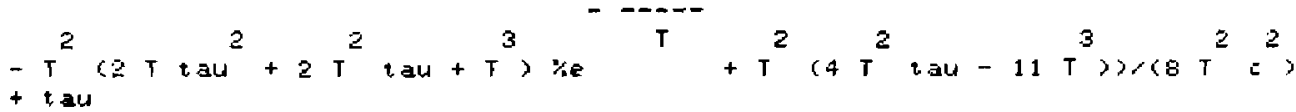

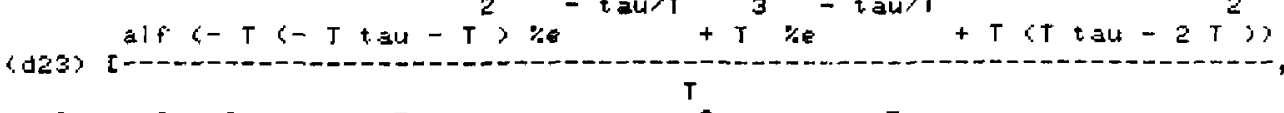

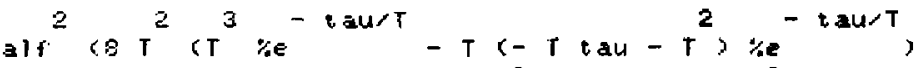

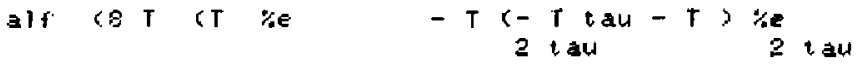

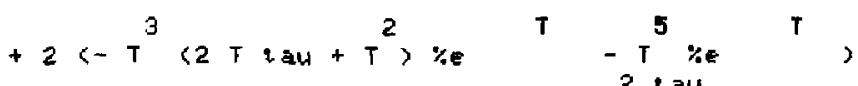

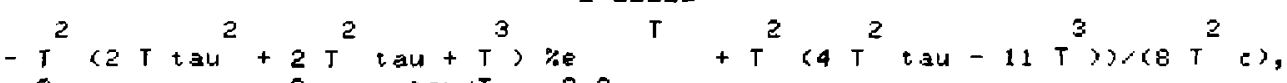

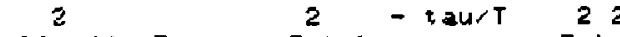

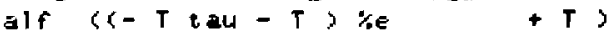




$$
\begin{aligned}
& 2 T^{2}
\end{aligned}
$$

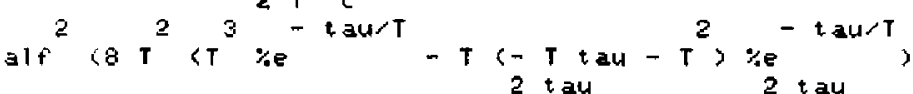

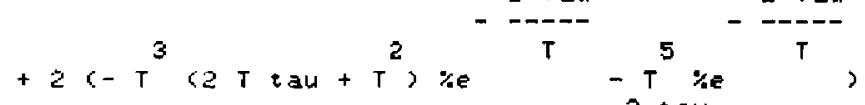

$$
\begin{aligned}
& 2 \text { tau }
\end{aligned}
$$

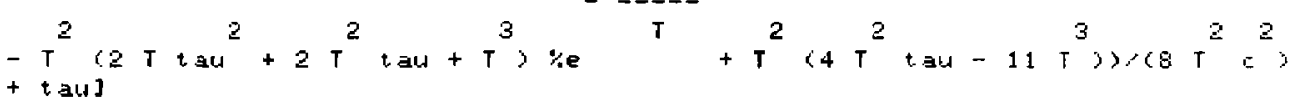

(c24) rel_chg_moj(gauss,tau);

$$
\begin{aligned}
& =
\end{aligned}
$$

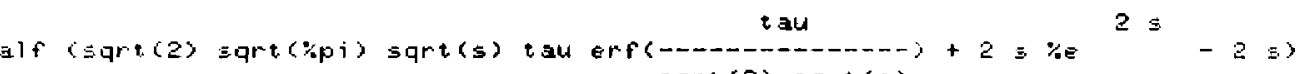

$$
\begin{aligned}
& \text { squt (2) } \operatorname{squ}(\xi)
\end{aligned}
$$$$
2
$$

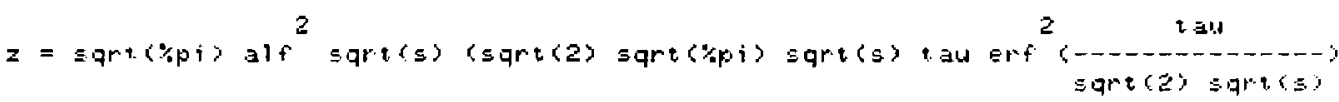

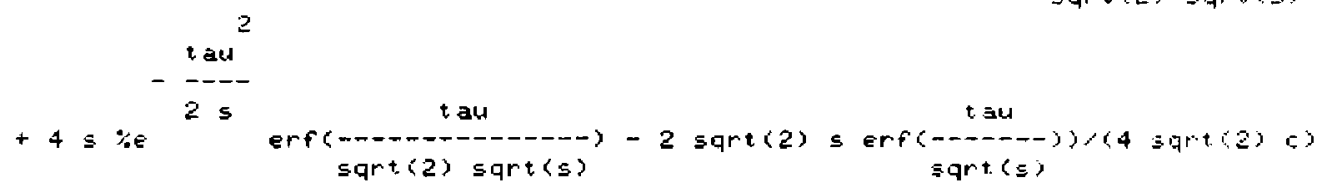

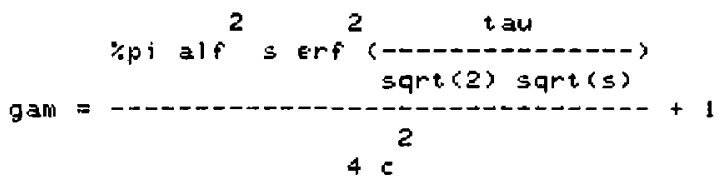

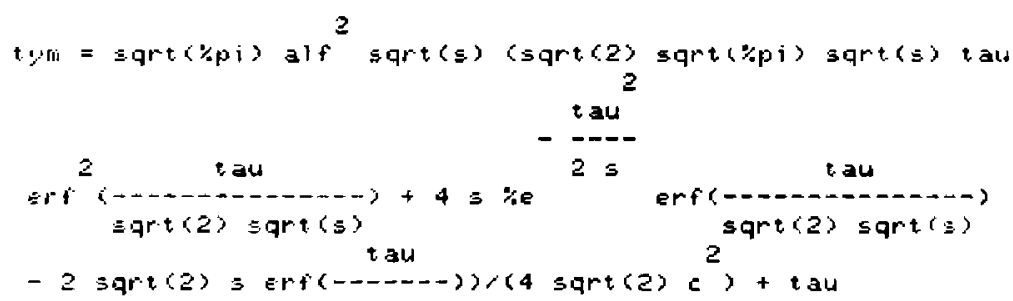$$
\text { squecs }(s)
$$

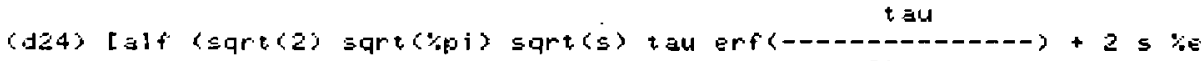

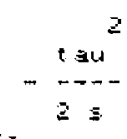

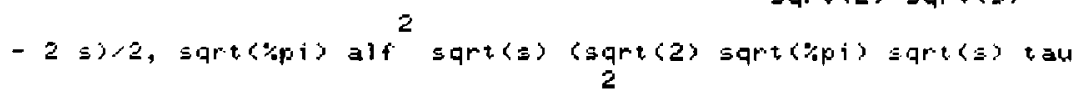

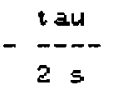




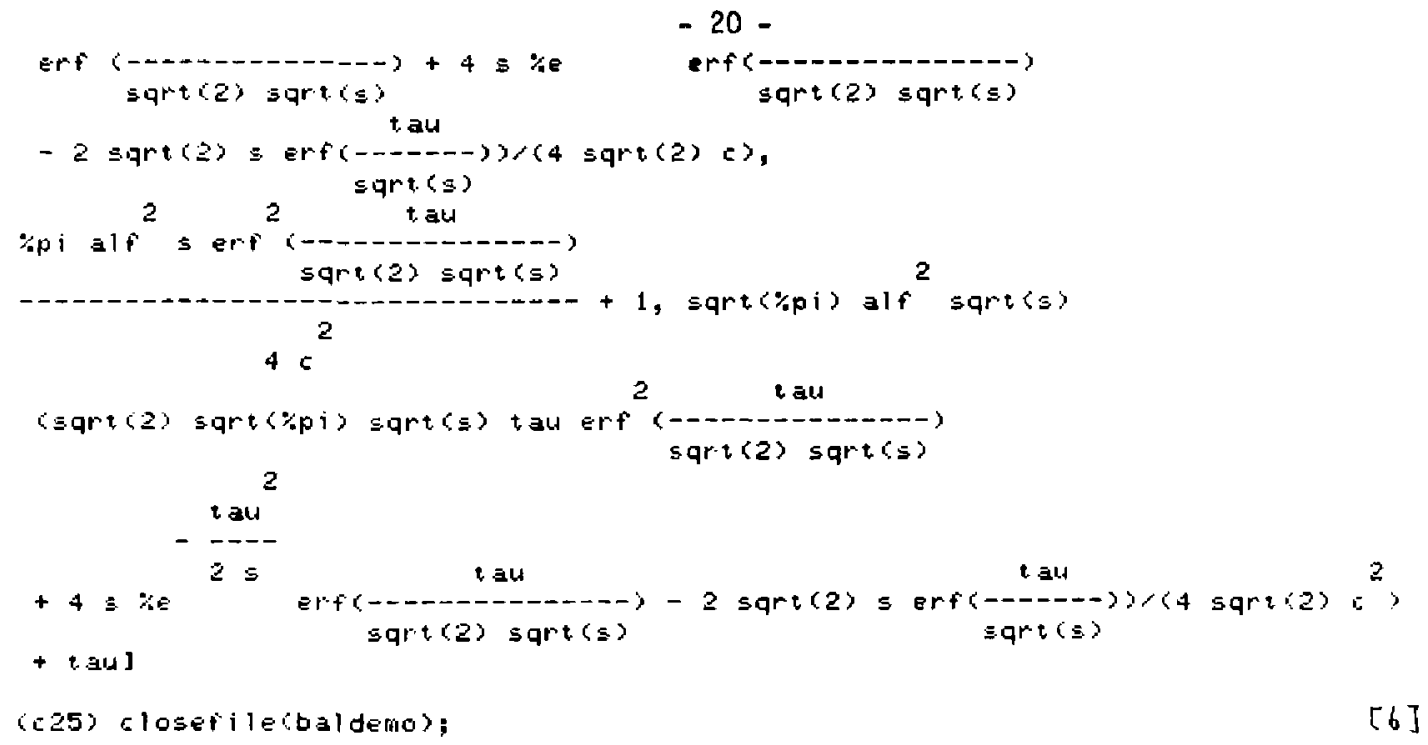




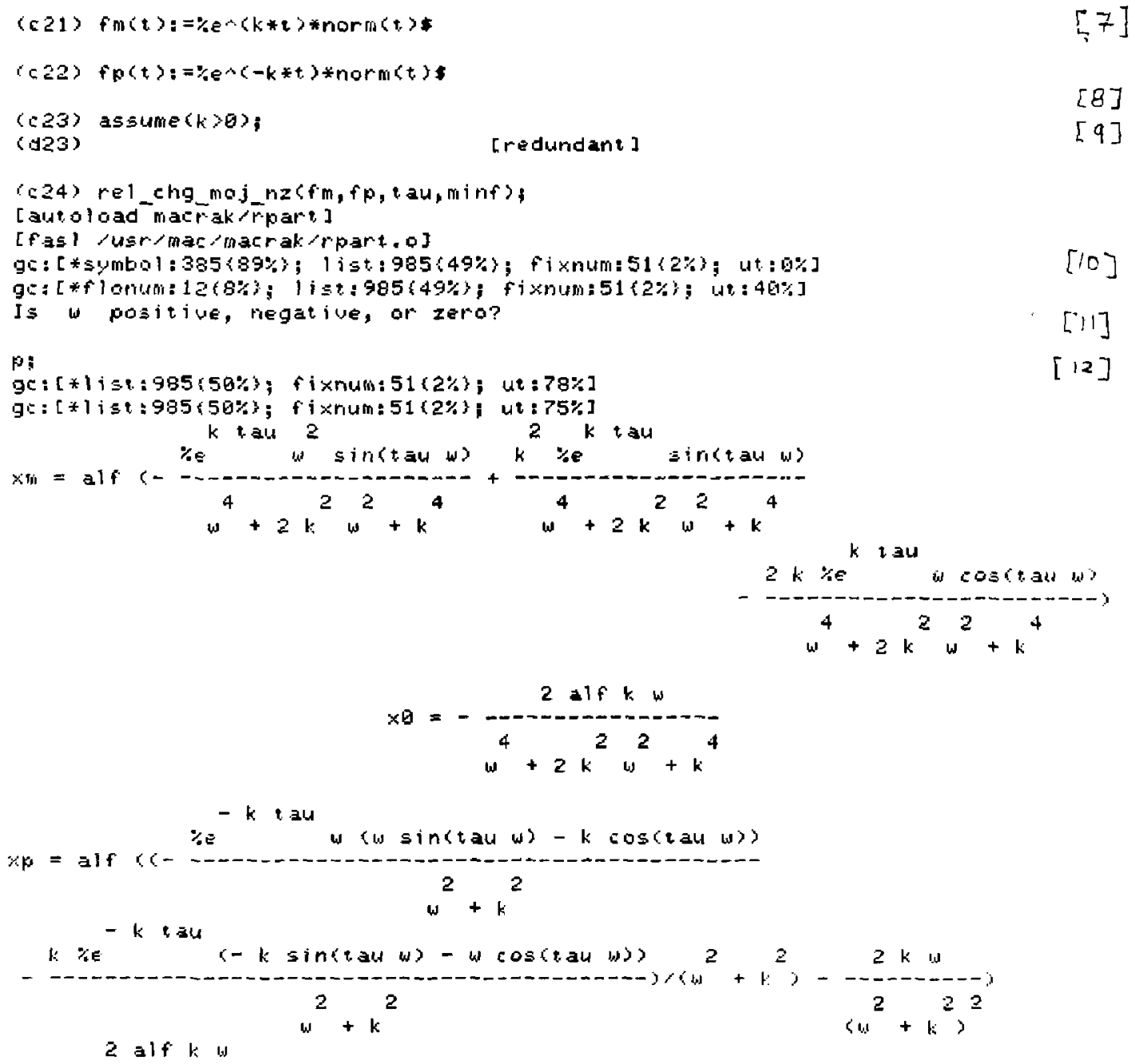


$w^{4}+2 k^{2} w^{2}+k^{4}$

$g<:[* 1 i \leq 1: 985(50 \%) ; f i \times n u m: 51(2 \%) ;$ ut: $73 \%]$ ge: [*1ist:985\{50\%\}; fixnum:51\{2\%); ut: $73 \%]$ ge: [*1ist: $985\{51 \%\}$; fixmum:51(2\%); ut: $75 \%]$

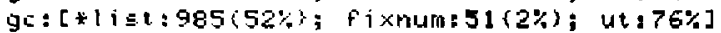
马с: $[* i$ i $\equiv t: 985(52 \%)$ fixnum:51(2\%); ue: $77 \%]$ gc: [*1 ist: $985(52 \%)$ fixnum:51(2\%); wr. $75 \%]$ gc: $[* 1$ ist: $985\{52 \%$; fixhum: $51\{2 \%$; ur: $71 \%]$ ge: $[* 1$ ist: $985\{51 \%)$ fixhum: $51\{2 \%\}$ ut: $73 \%]$ gc: [*1 ist:985\{51\%\}; fixnum:51(2\%); wt:71\%]

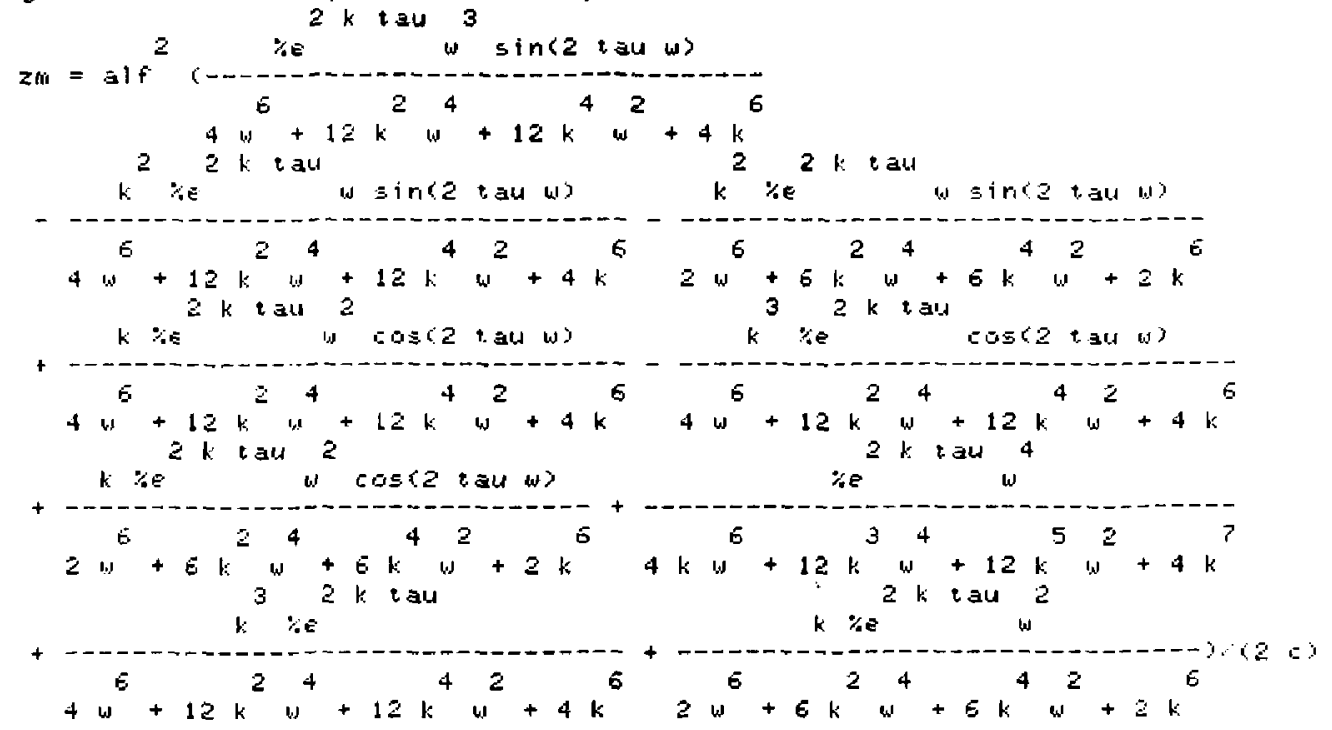
$20=31 f^{2}$

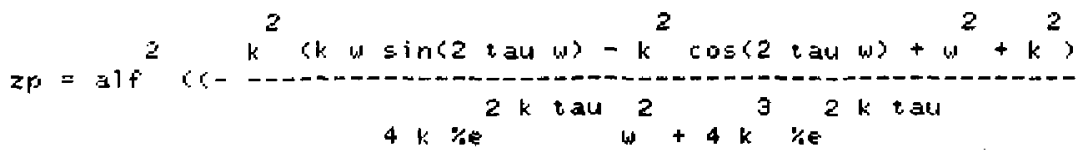
$2 \quad 2 \quad 2 \quad 2$ w $(k w \sin (2 \tan w)-k \cos (2 \tan w\rangle-w-k)$ $4 k \% e^{2 k} \mathrm{k} a \mathrm{w}^{2}+4 k^{3} 2 k$ iau

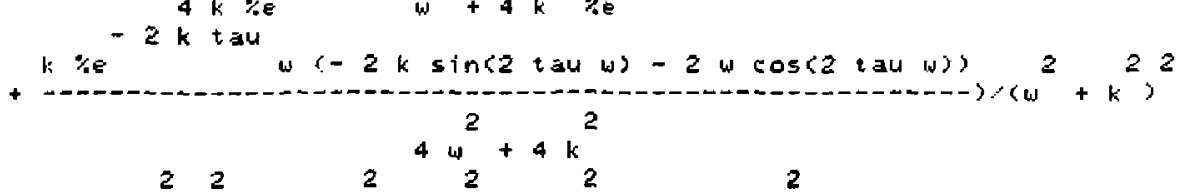




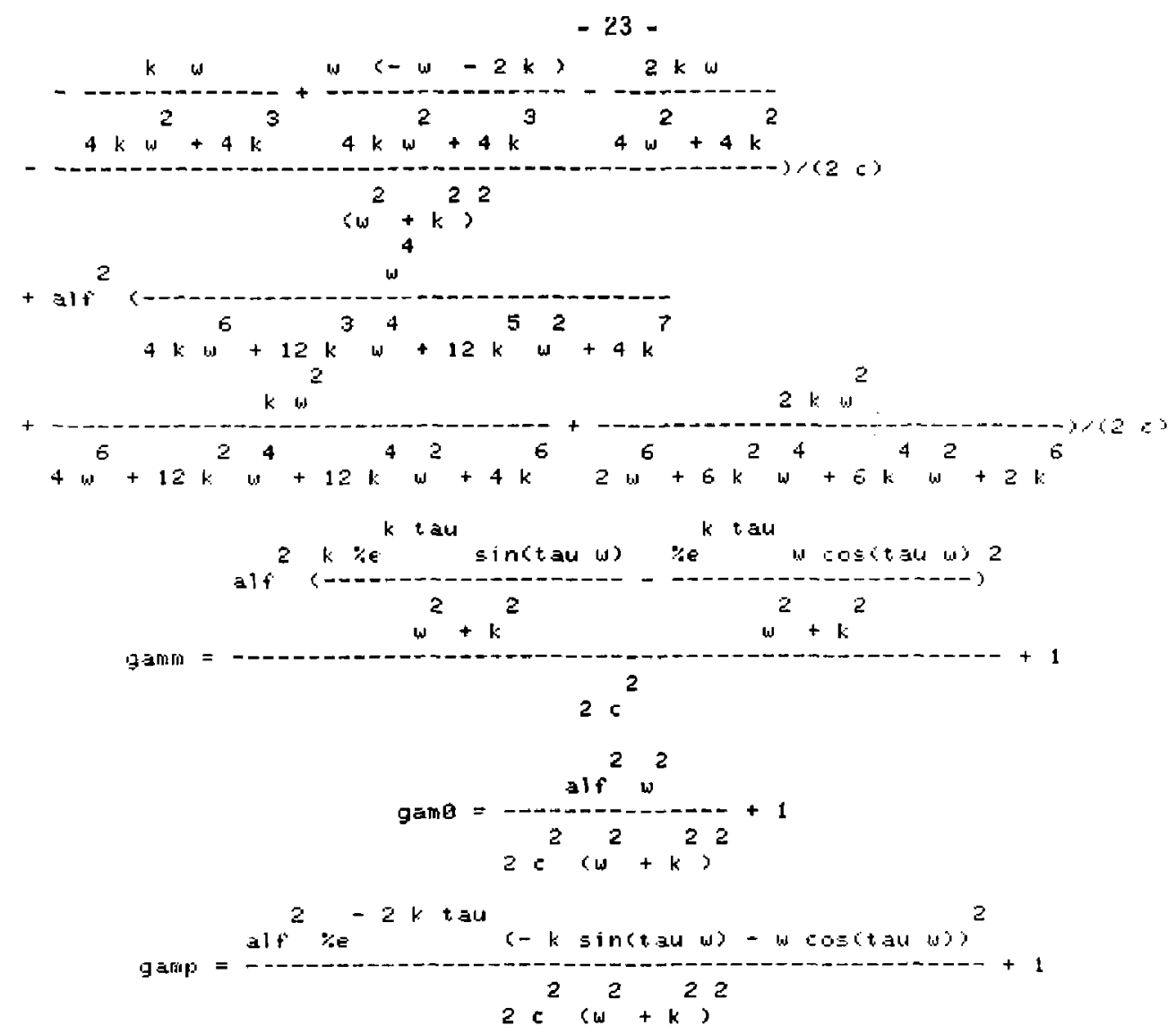

$g \subset:\{* \mid$ ist:985\{52\%\}; fixnum:51\{2\%\}; we:73\%]

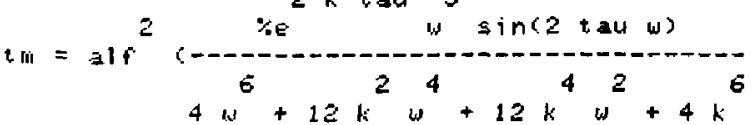

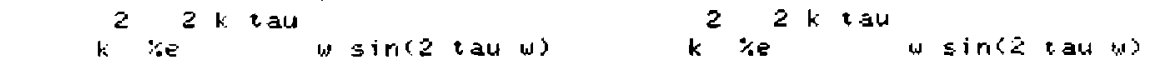

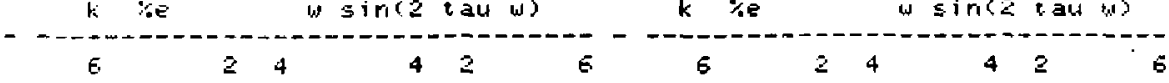

$$
\begin{aligned}
& 4 u+121 w+12 k w+4 k w+2 w+6 k w+6 k w+2 k \\
& 2 k+a k 2
\end{aligned}
$$

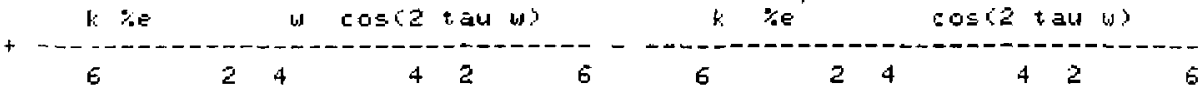

$$
\begin{aligned}
& 4 w+12 k w+12 k w+4 k+4 w+12 k w+12 k w+4 k \\
& 2 k \text { tau } 2 \text { a } 2 \text { tau } 4 \\
& 2 w^{6}+6 k^{2} w^{4}+6 k^{4} w^{2}+2 k^{6} 4 k w^{6}+12 k^{3} w^{4}+12 k^{5} w^{2}+4 k^{7} \\
& 3 k^{2 k t a u} k \text { ise }{ }^{2 k} \text { ian } 2 \\
& 4 w^{6}+12 k^{2} \omega^{4}+12 k^{4} w^{2}+4 k^{6} 2 w^{6}+6 k^{2} w^{4}+6 k^{2}+2 k k^{2}+2,
\end{aligned}
$$




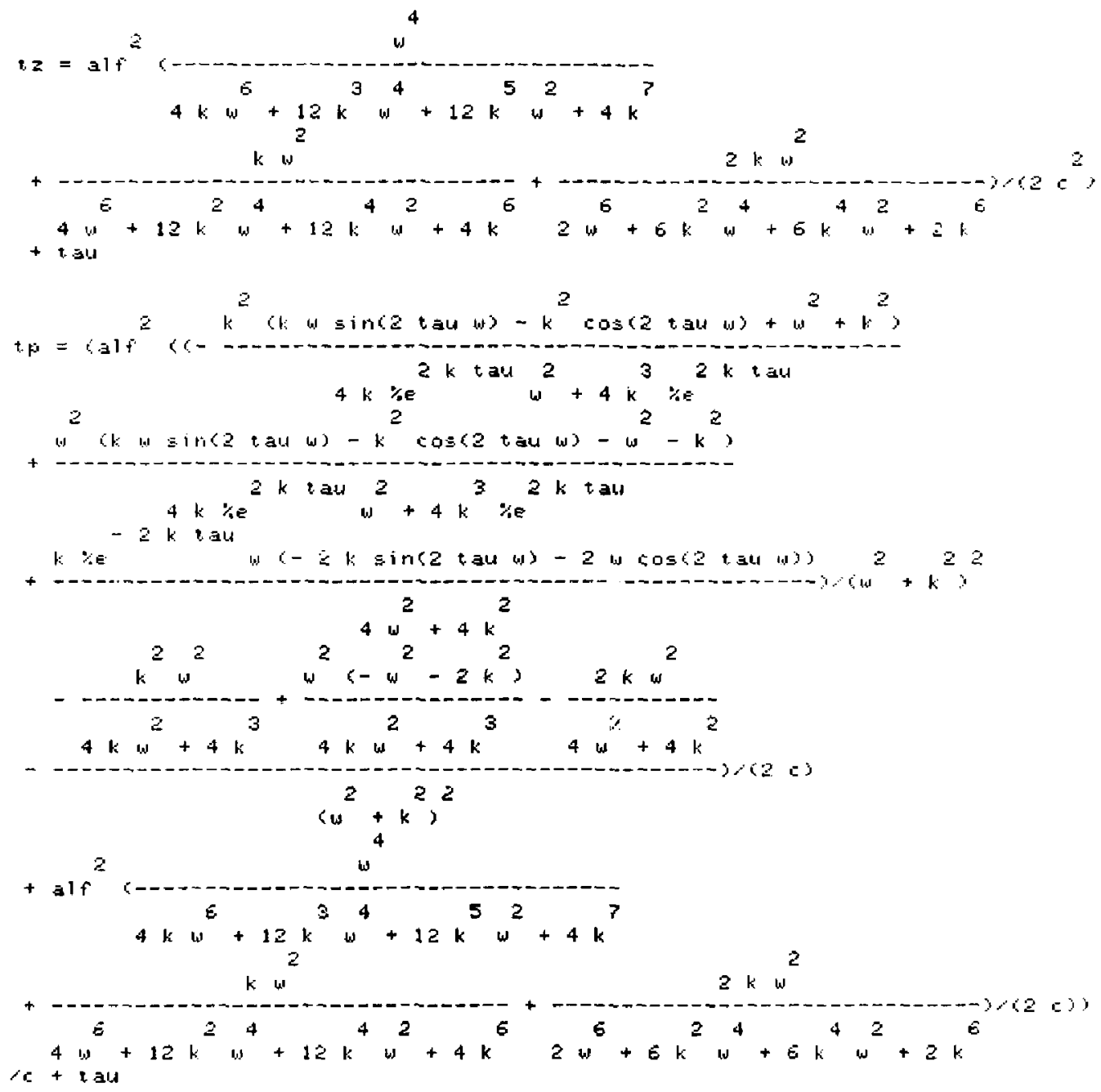

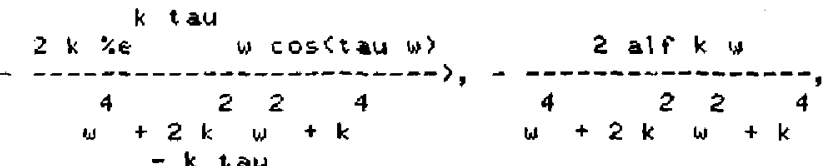

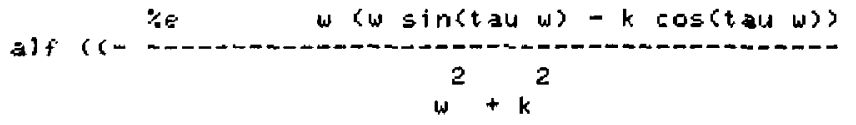$$
\% e^{k \operatorname{tas} w^{2}} \sin \langle t a w \omega\rangle k^{2} \% e^{k \operatorname{tau}} \sin (t a u w\rangle
$$

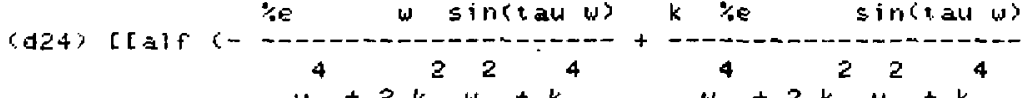

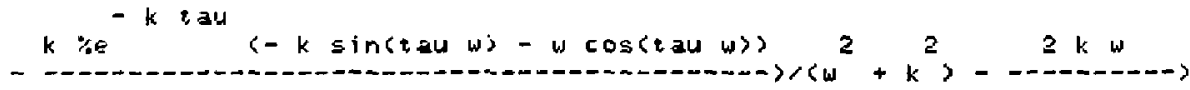




$$
\begin{aligned}
& w^{2}+k^{2} \quad 2 k \text { tau } 3 \quad\left(w^{2}+k^{2}\right)^{2} \\
& \begin{array}{c}
2 \text { alf } k w \\
4+2 k^{2}{ }^{2}+k^{4}
\end{array} \\
& 22 k \text { a a } 24 w+12 k \begin{array}{c}
4 w \\
2 k \text { tau }
\end{array}
\end{aligned}
$$

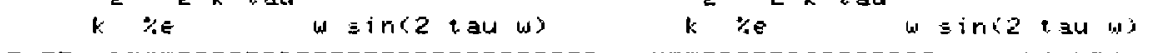

$$
\begin{aligned}
& 4 u^{6}+12 k^{2} 4+12 k^{4} w^{2}+4 k^{6} 2 w^{6}+6 k^{2} w^{4}+6 k^{2}+2 k \\
& 2 k \text { rau } 2 \text { is } 2 k t a u
\end{aligned}
$$

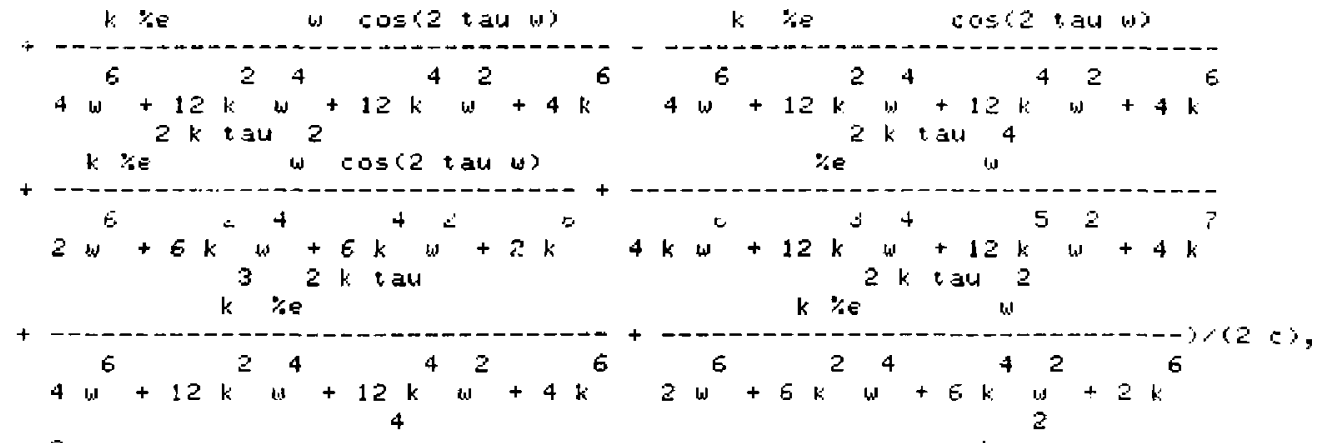

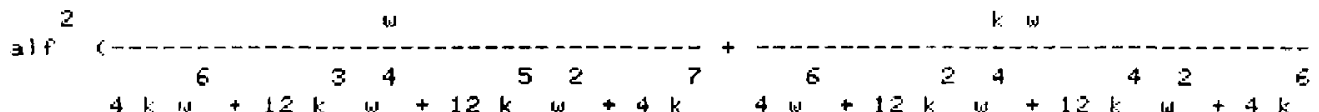

$$
\begin{aligned}
& 4 k^{6}+12 k^{3} w^{4}+12 k^{5} w^{2}+4 k^{7} 4 w^{6}+12 k^{2} u^{4}+12 k^{2} w^{2}+4 k^{5} \\
& 2 w^{6}+6 k^{2} w^{4}+6 k^{4} w^{2}+2 k^{6} \\
& { }^{2}\left(k^{2}(k-1) \sin (2 \sin w)-k^{2} \cos (2 \tan w)+w^{2}+k^{2}\right)
\end{aligned}
$$

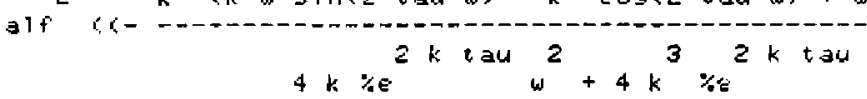

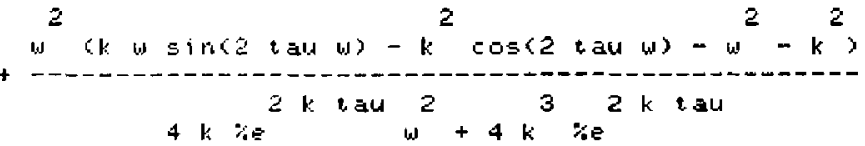

$$
\begin{aligned}
& g=:[* 1 \text { i:t: } 985(53 \%) ; \text { fixnom: } 51\{2 \%) ; \text { ut: } 70 \%] \\
& -2 k \text { e su }
\end{aligned}
$$

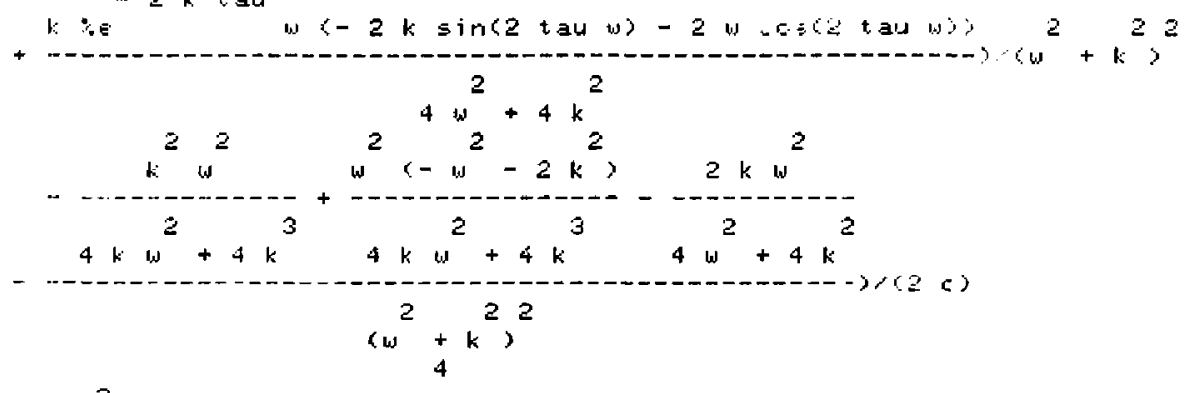

$$
\begin{aligned}
& +\operatorname{alf}^{2}\left(-6 \mathrm{w}^{6}+12 \mathrm{k}^{3} \mathrm{w}^{4}+12 k^{5} \mathrm{w}^{2}+4 k^{7}\right.
\end{aligned}
$$




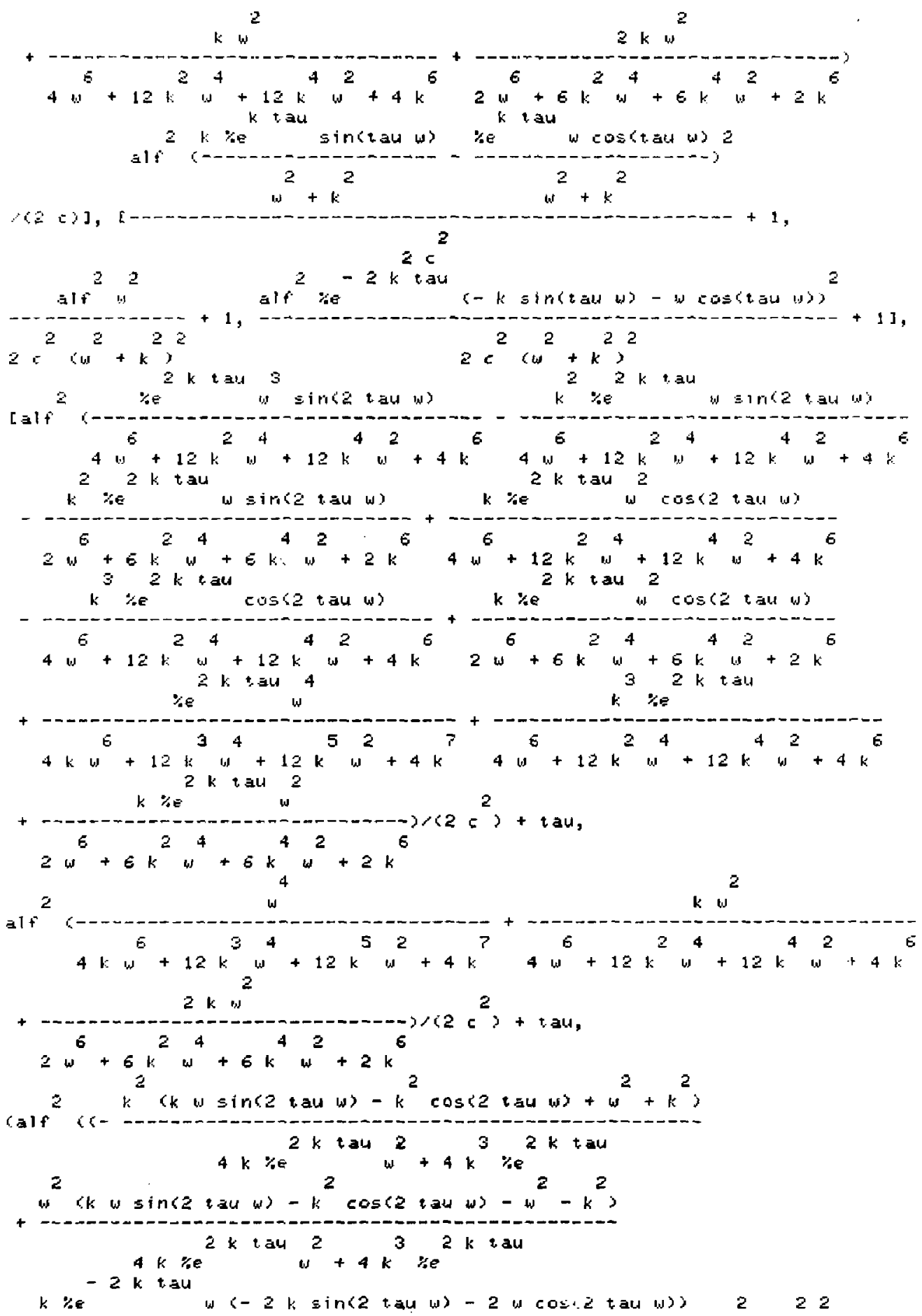




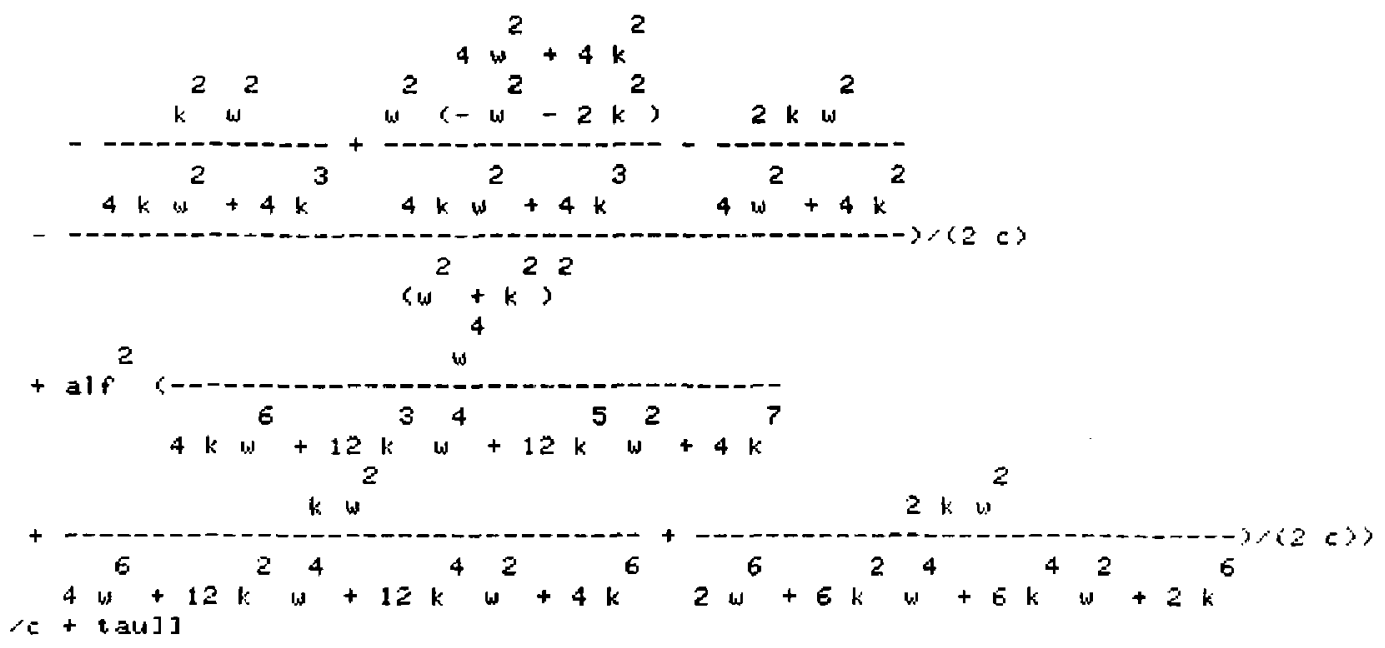




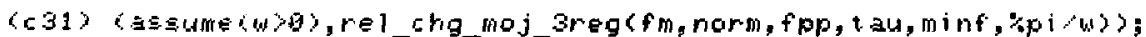

$g c:[\% 1$ ist: $985\{52 \%\} ;$ fi $x$ num: $51\{\overline{2} \%\}$ ut: $86 \%]$

gc: [*1 ist: $985\{52 \%\}$ fixnum: $51\{2 \%\}$; ut: $74 \%]$

gc: [*ist: $985\{53 \%\rangle$ fixnum:51\{2\%\}; ut: $73 \%]$

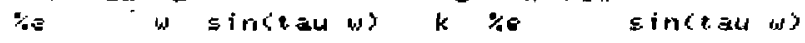




$$
\begin{aligned}
& x \mathrm{n}=\mathrm{alf}\left(\mathrm{w}+2 k^{2} \mathrm{w}^{2}+k^{4} w^{4}+2 k^{2} w^{2}+k^{4}\right. \\
& 2 k \% e^{k \text { tau }} w \cos (t a u w) \\
& 42 \mathrm{2} 4 \\
& w+2 k+k \\
& x \theta=-\frac{2 a l f k w}{4}+2 k^{2} w^{2}+k^{4}
\end{aligned}
$$

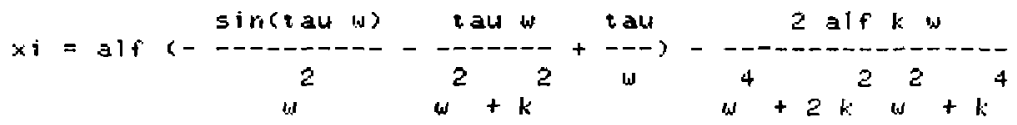

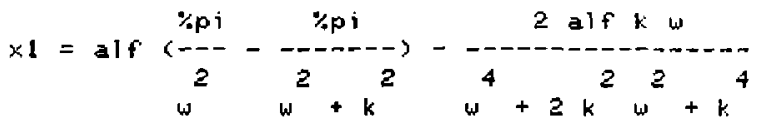$$
\text { - k tat }
$$

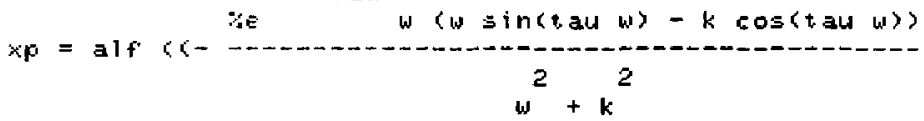$$
\text { - }
$$$$
1.1
$$

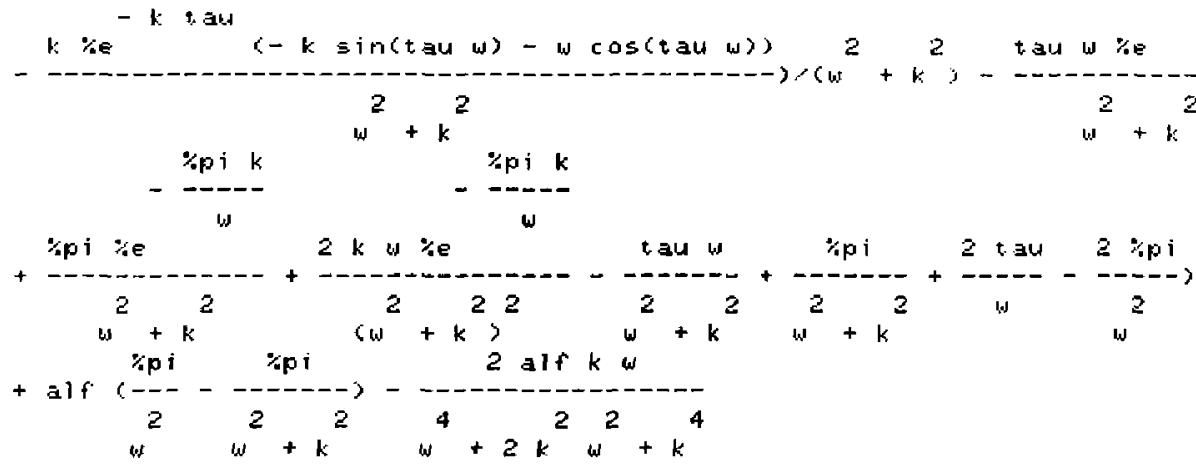

gC: [*1; $3 t: 985\{5: 3 \%\} ;$ fixnum: $51\{2 \%\}$ ut: $74 \%]$

gc: $[\because 1 i \leq t: 985\{54 \%\} ; j$ f

$g c:[* 1 j \leq t: 985(54 \%) ;$ fixnm: $51(2 \%) ;$ ut: $71 \%]$

g5: [*1i $\leqslant t: 985\{54 \%\} ; f i x n u m: 51\{2 \%\} ; \quad$ t: $74 \%]$

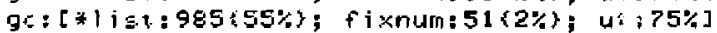

gE: [*1i $\equiv 2: 985(55 \%) ;$ fxnum:51\{2\%); ut:73\%]

gr: [*1i $[* 1,985(55 \%) ;$ fixnum:51(2\%); wt:76\%]

$95:[* 1 i \equiv t: 985\{54 \%\} ;$ fixnum:51\{2\%; we: $73 \%]$

$g 5:[* 1 \mathrm{i} \equiv 2: 985\{55 \%\} ; f i \times n u m: 51(2 \%) ;$ wR: $71 \%]$

gc: [*1ist:985<55\%; fixnum:51\{2\%) ut:?1\%]

$g c:[* 1$ ist:985\{55\%\}; fixnum:51\{2\%\} ut:?3\%]

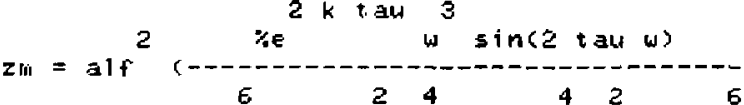

$$
\begin{aligned}
& 4 w^{2}+12 k^{2} w^{4}+12 k^{2} w^{2}+4 k \\
& k^{2} * e^{2 k \operatorname{tau}} \omega \sin (2 t \text { aw } w) \quad k^{2} x e^{2 k t a u} w \sin (2 t a u w)
\end{aligned}
$$




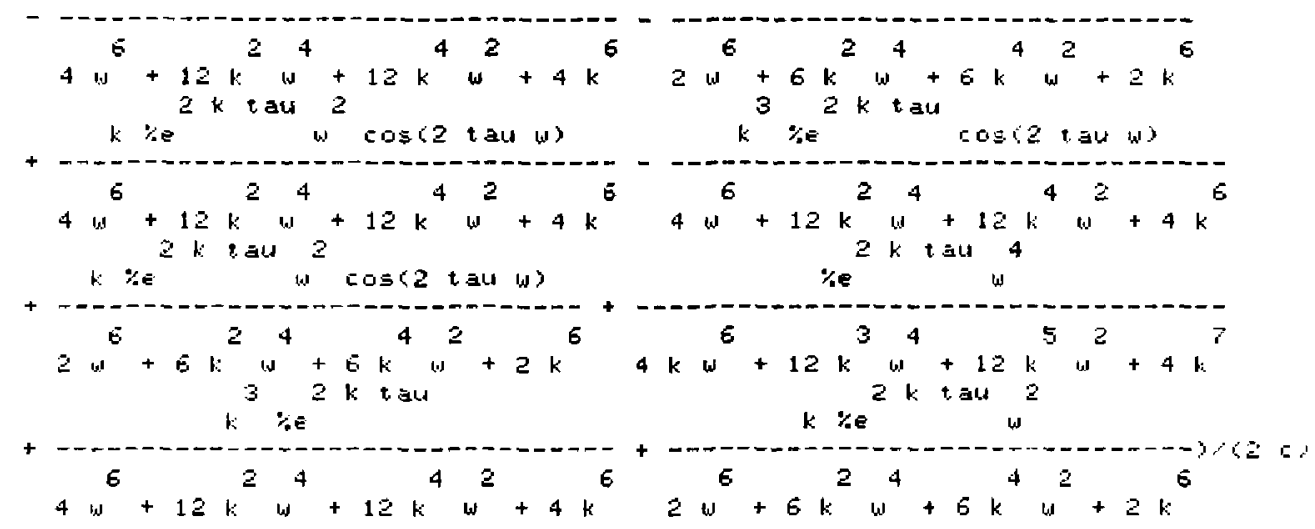

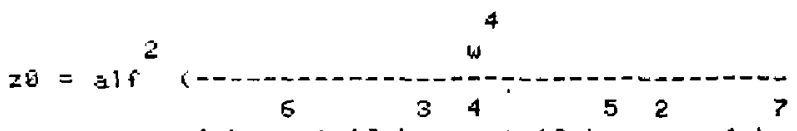

$$
\begin{aligned}
& 4 k w^{5}+12 k^{3}+12 k^{5} w^{2}+4 k \\
& 2
\end{aligned}
$$

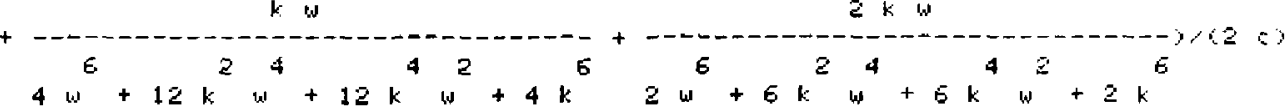

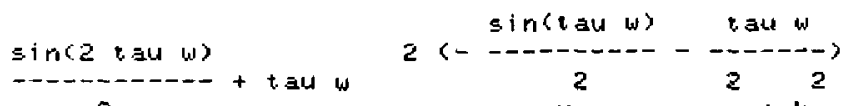

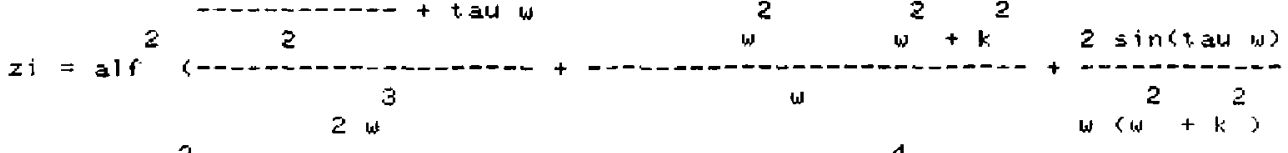

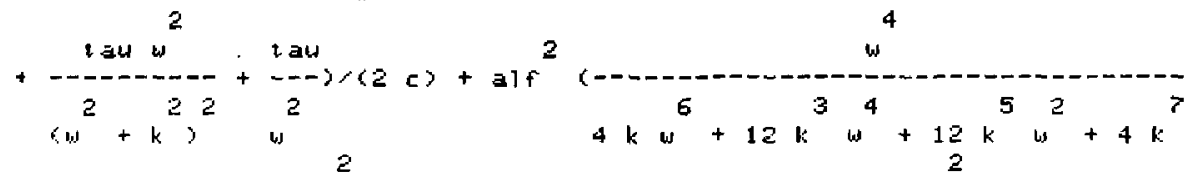

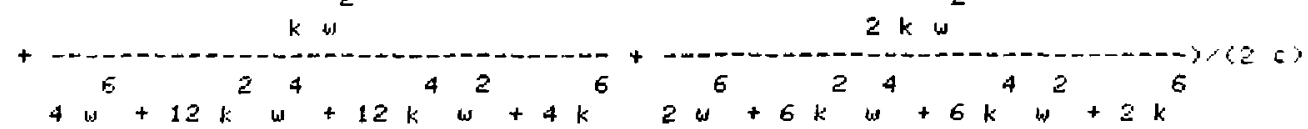

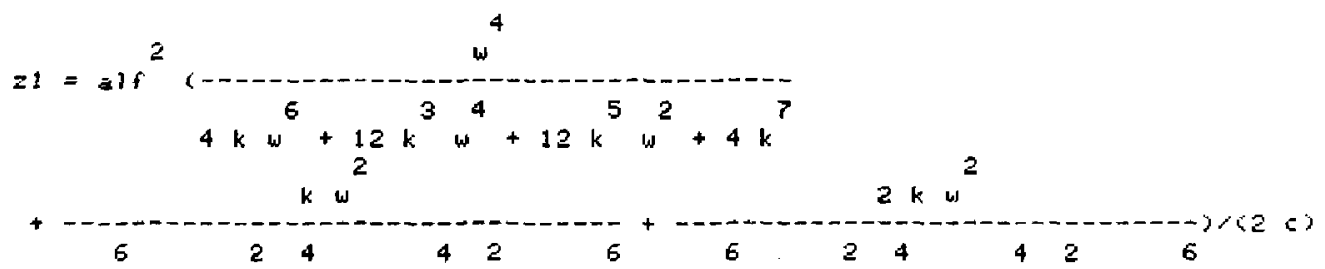

$$
\begin{aligned}
& 4 w^{6}+12 k^{2} w^{4}+12 k^{4} w^{2}+4 k^{6} w^{6}+6 k^{2} w^{4}+k^{4}+2 w^{2} \\
& \operatorname{alf}^{2}\left(-\frac{2 \mathrm{kpi}}{2} \frac{\mathrm{kpi} w}{2}+\frac{3 \mathrm{kpi}}{22}+\frac{3}{3}\right. \\
& \text { w } x w+k)(w+k) 2 w \\
& 2 c
\end{aligned}
$$

gc: [*1 ist: $985(57 \%)$ fixnum: $51\{2 \%$ ) ut:72\%] 


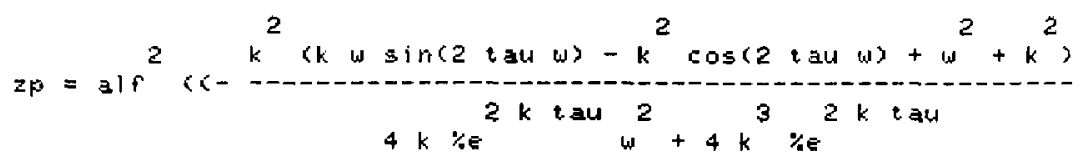

$$
\begin{aligned}
& w^{2}\left(k w \sin \langle 2 \tan w\rangle-k^{2} \cos (2 t a w w)-w^{2}-k^{2}\right\rangle
\end{aligned}
$$

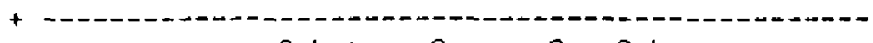

$$
\begin{aligned}
& 4 k \% e^{2 k i a w} w^{2}+4 k^{3} \% e^{2 k t a u} \\
& -2 k t \text { a }
\end{aligned}
$$

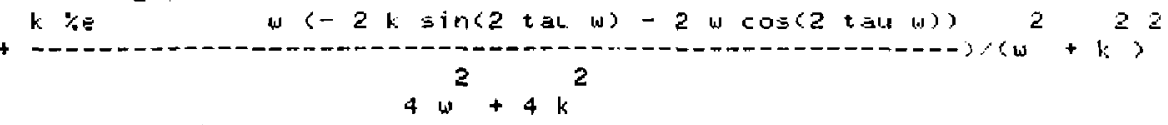

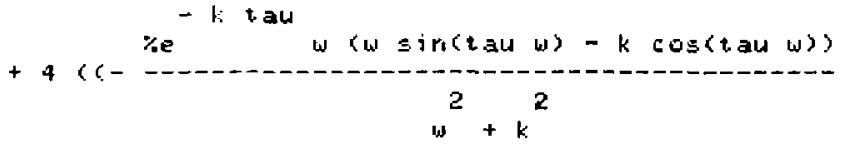

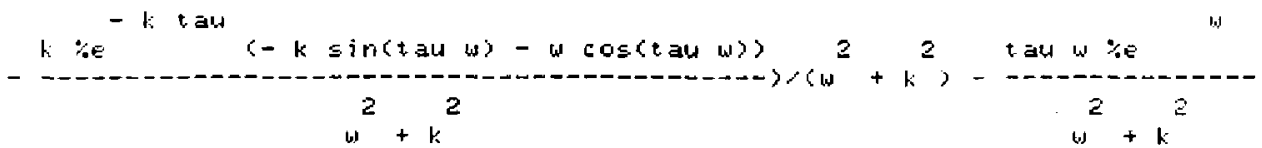

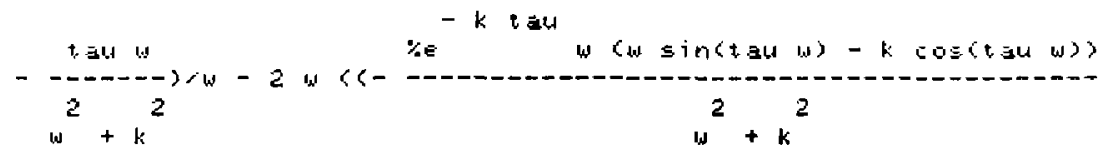

$$
\begin{aligned}
& \text { ipi } \mathbf{k}
\end{aligned}
$$$$
\text { - } k \text { tau }
$$

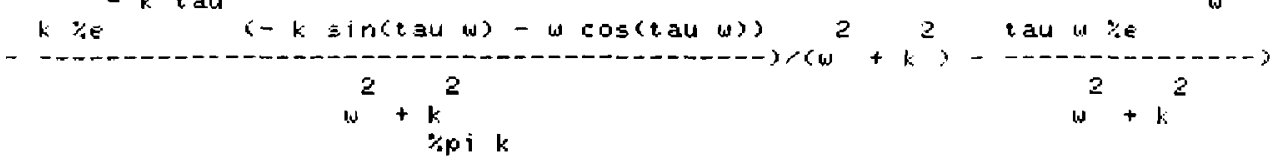$$
\frac{7 p i k}{w}
$$

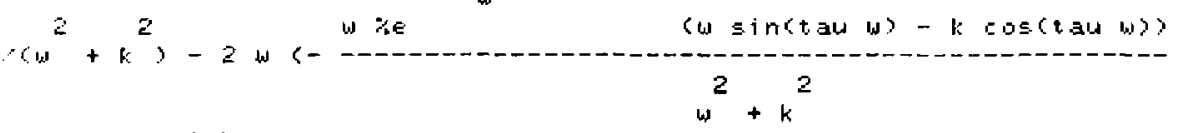$$
-\frac{\pi p i k}{w}
$$

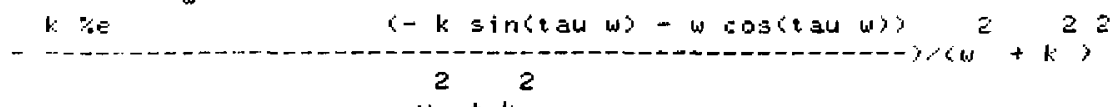

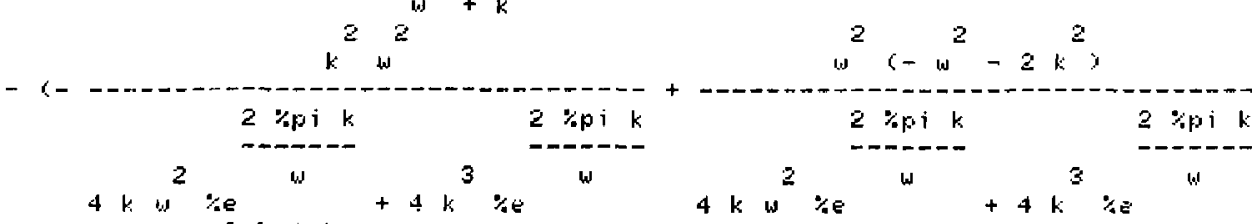

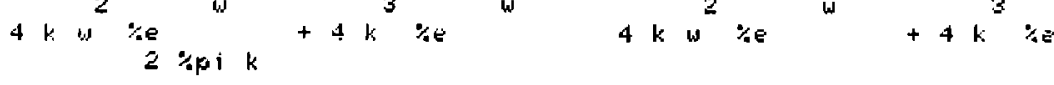$$
\text { b }
$$

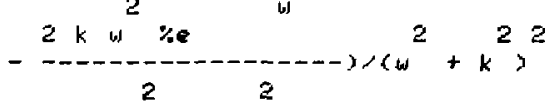




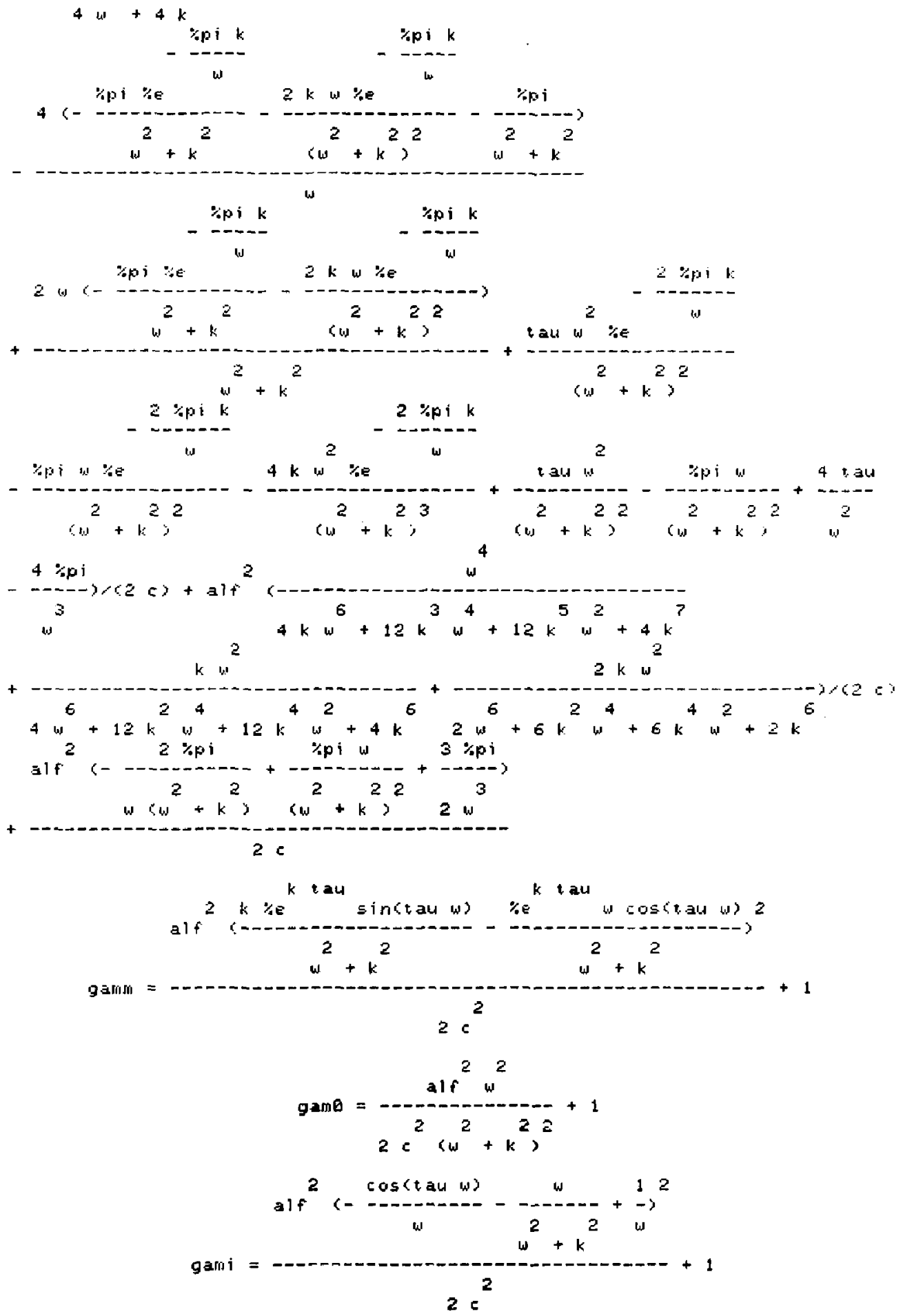




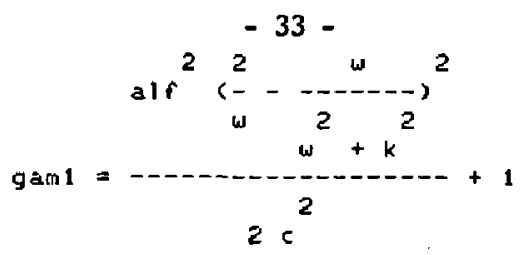

gamp $=$
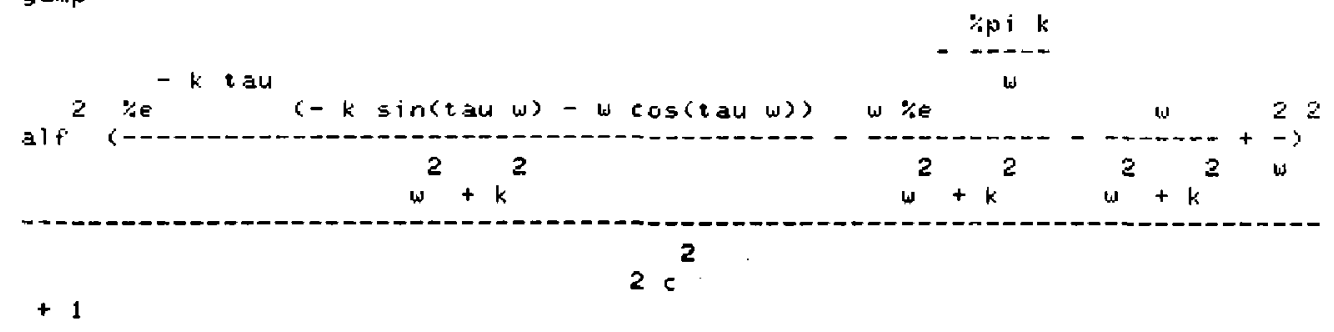

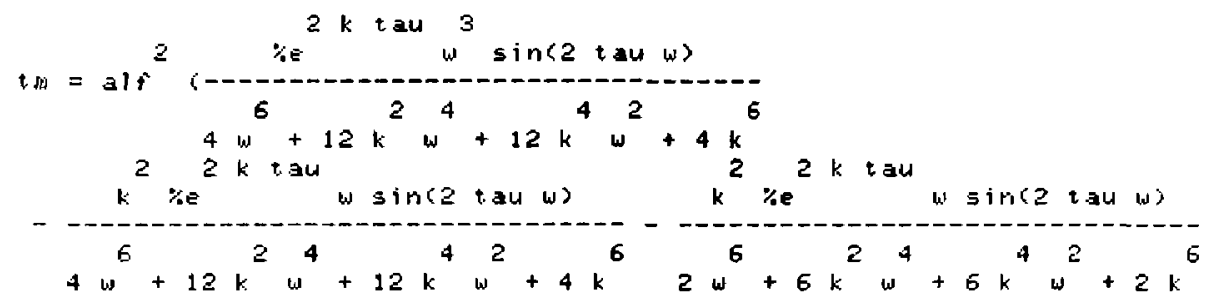

gc: [*1ist: $985\{58 \%\} ;$ fixnum: $51(2 \%) ;$ ut:67\%]

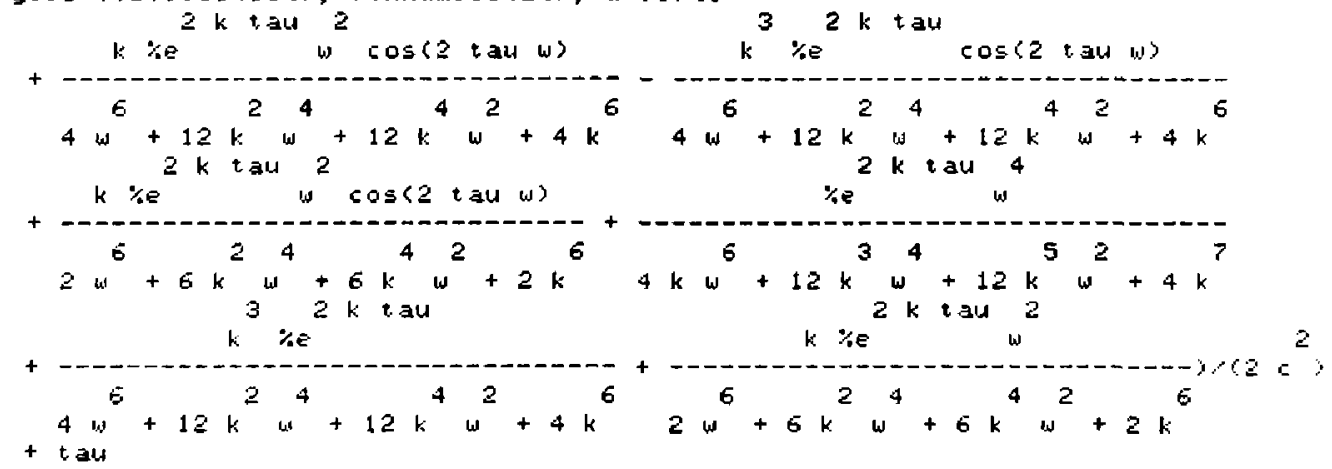

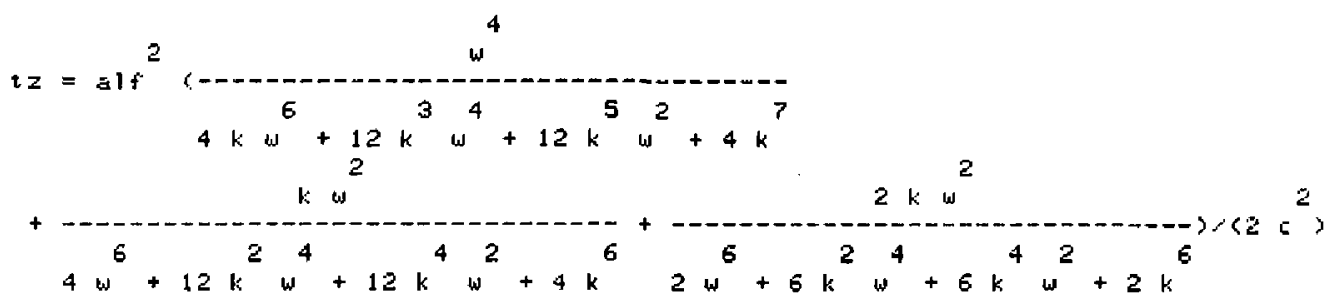

$+t$ aw

$$
\begin{aligned}
& \sin (2 \text { tau } w) \\
& 2 \\
& \frac{1}{2} \\
& 2\left\langle-\frac{\sin (t a u w)}{2} w^{2}+k^{2}\right.
\end{aligned}
$$


$j=\langle a l i$

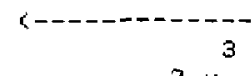

$2 w$

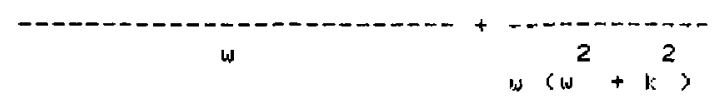

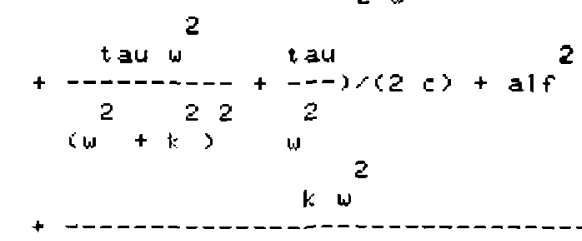

$4 w^{6}+12 k^{2} w^{4}+12 k^{4} w^{2}+4 k^{6}$

c + t-at

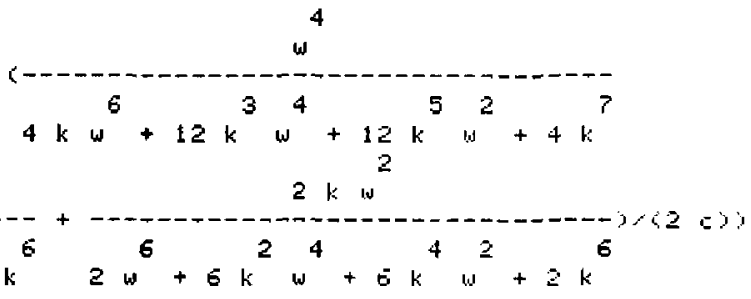

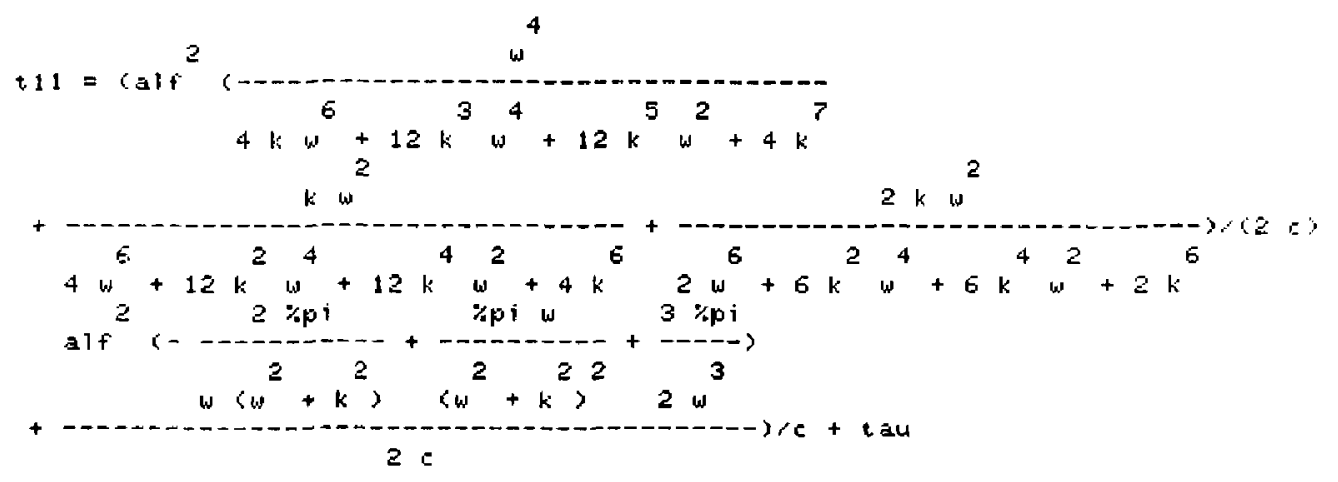

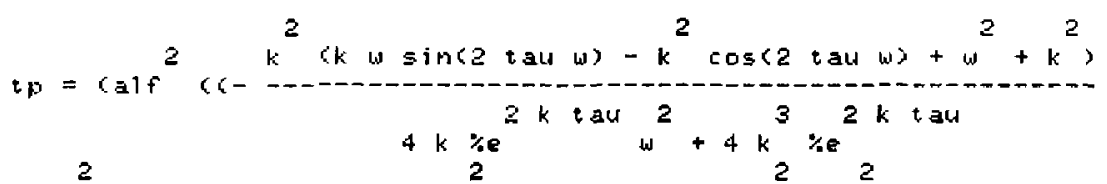

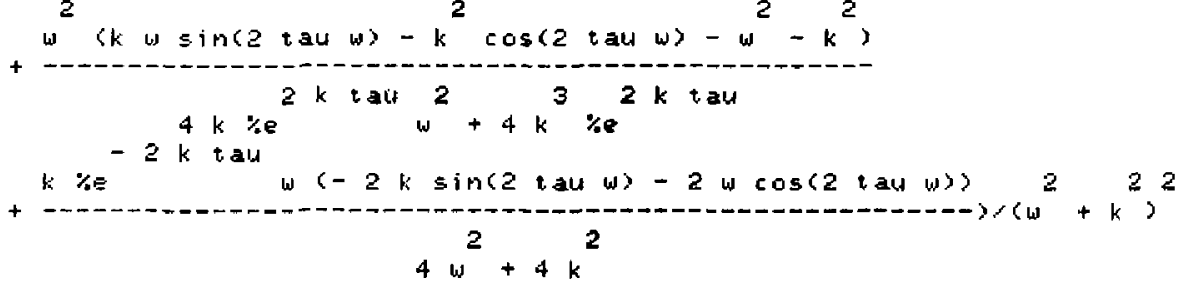

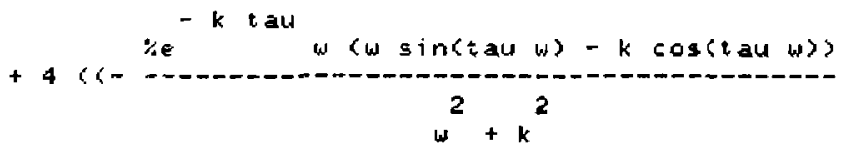

xpik

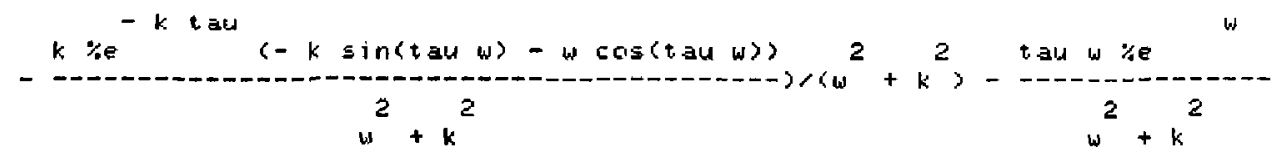

ge: [*1 ist: $995\{64 \%\}$; fixnum:51\{2\%), ut: $66 \%]$

$-k+a u$

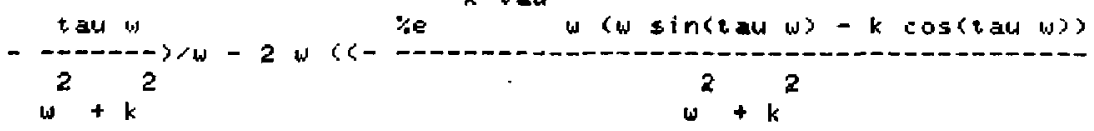

xpi $k$ 


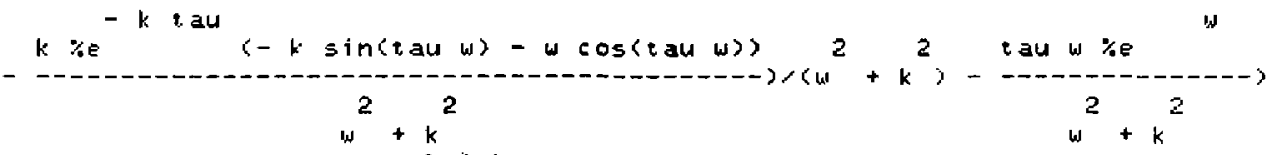

$$
\begin{aligned}
& \text { \%pik } k \text { taw }
\end{aligned}
$$

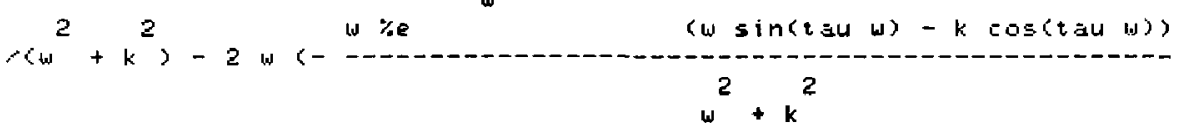

$$
\begin{aligned}
& \text { rpik } \\
& k \text { \%e }(-k \sin (t a u w)-w \cos (t a w w)\rangle \quad 2+2
\end{aligned}
$$

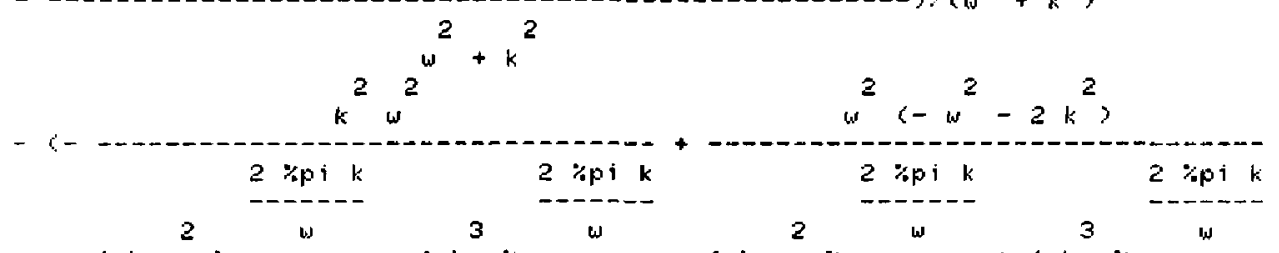

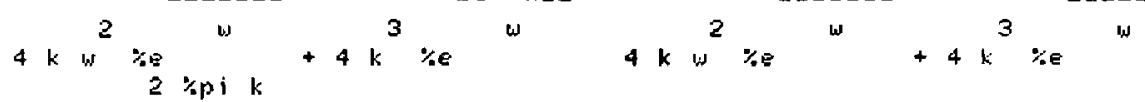

$$
\begin{aligned}
& -0 .-1 \\
& -\frac{2 k w^{2} z e}{4 w^{2}+4 k^{2}}
\end{aligned}
$$

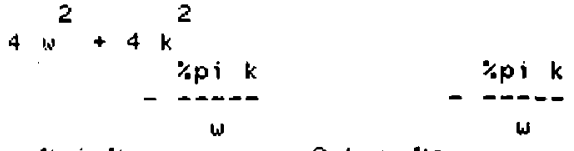

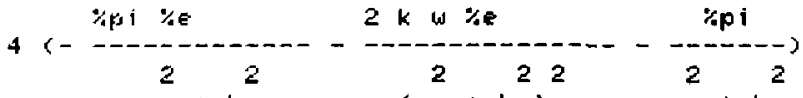

$$
\begin{aligned}
& w+k \quad(w+k)^{2} w+k \\
& \text { צip } k \text { d } k
\end{aligned}
$$

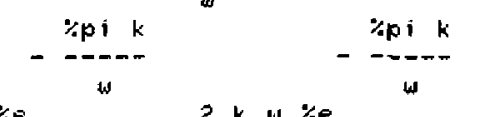

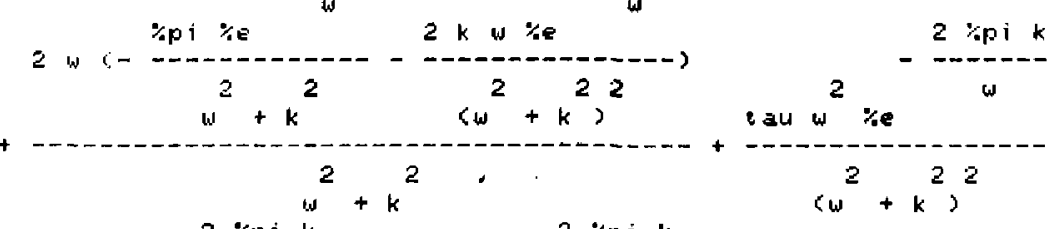

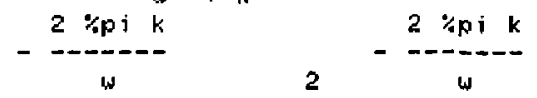

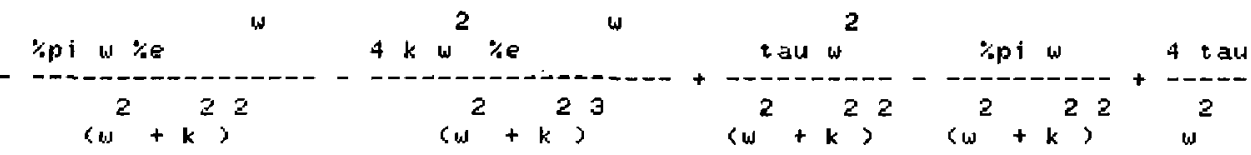

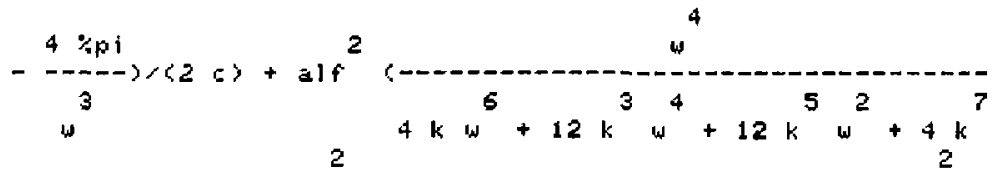


kw

$2 k w$

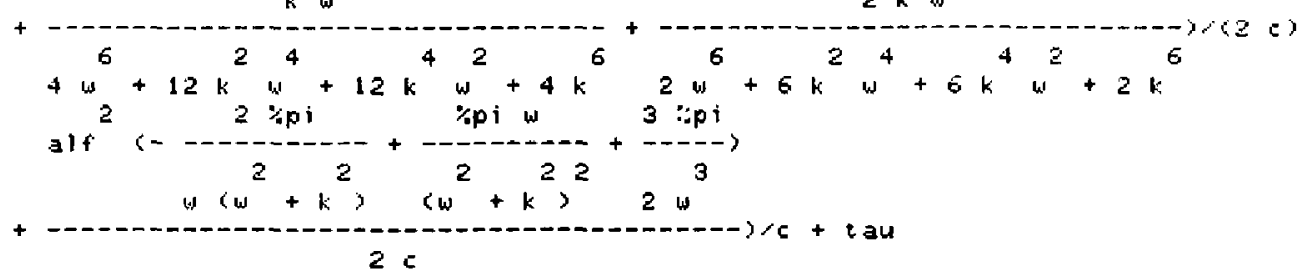

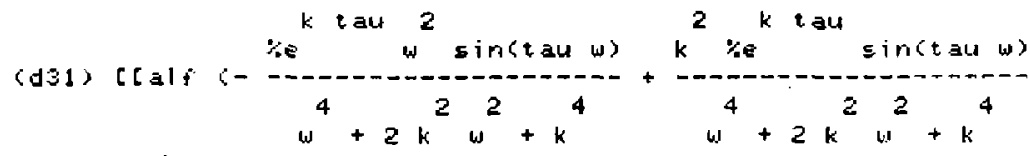

$$
\begin{aligned}
& \text { k tau }
\end{aligned}
$$

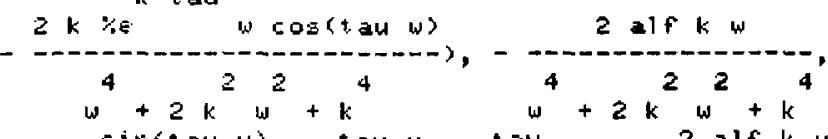

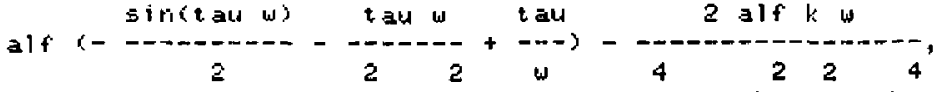

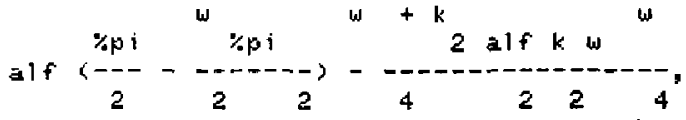

$$
\begin{aligned}
& w+k+2 w^{2} w^{2}+k \\
& \text { - } k t a u
\end{aligned}
$$

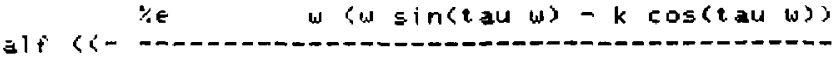$$
w^{2}+k^{2}
$$

$$
\begin{aligned}
& \text { - k tau }
\end{aligned}
$$

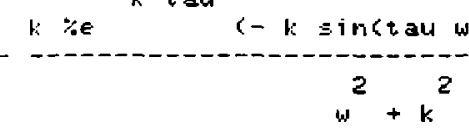

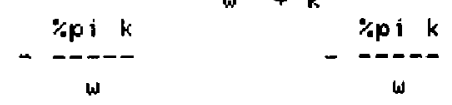

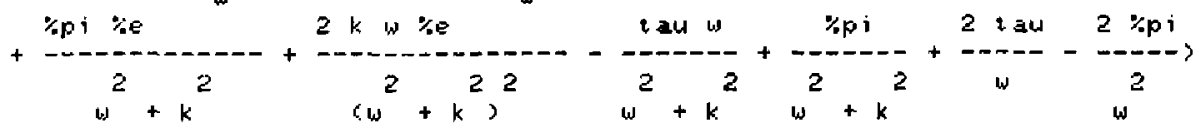

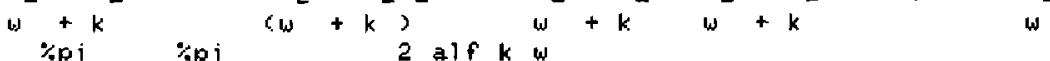

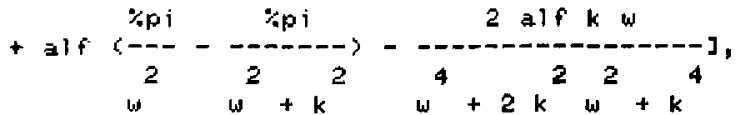

$$
\begin{aligned}
& \text { wi } 2^{w} k^{2}+k^{2} w^{4}+2 k^{2} w^{2}+k^{4} 22 k t a w
\end{aligned}
$$

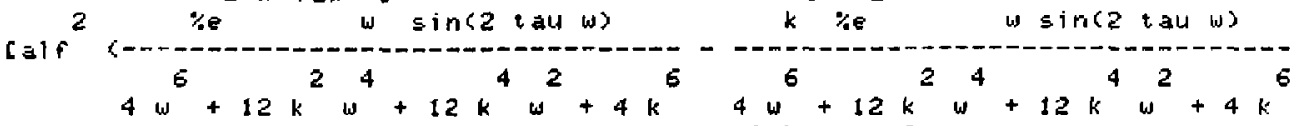

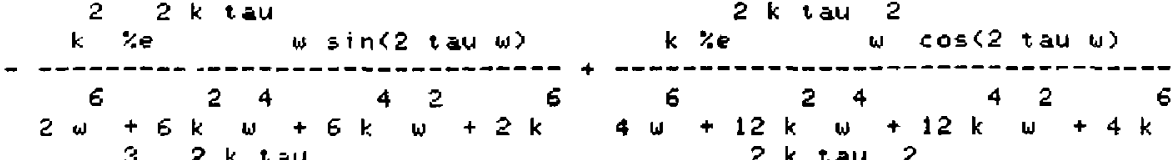$$
k \% e^{2} \cos (2 \tan \omega) \quad k \% e \quad \omega \cos (2 \operatorname{tat} w)
$$$$
\begin{array}{lllllllll}
6 & 2 & 4 & 6 & 6 & 2 & 4 & 4 & 2
\end{array}
$$ 


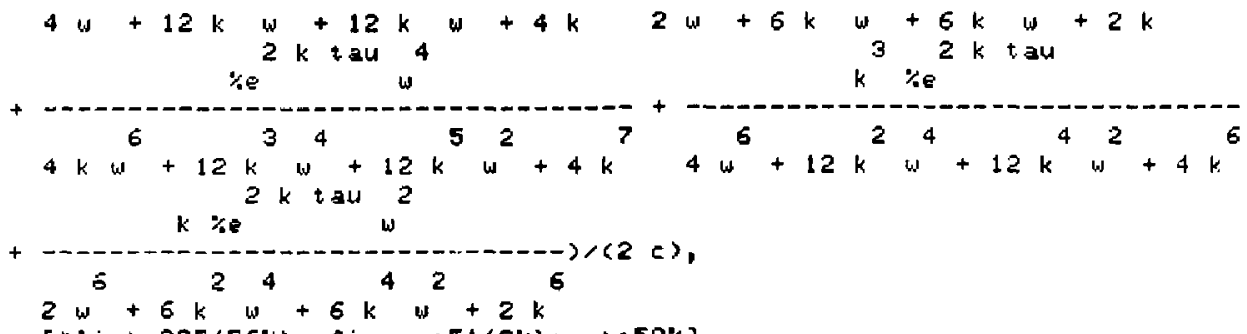

$g c:[* 1$ ist: $995\langle 56 \%) ; f i x n u m: 51(2 \%)$; wt: $59 \%]$

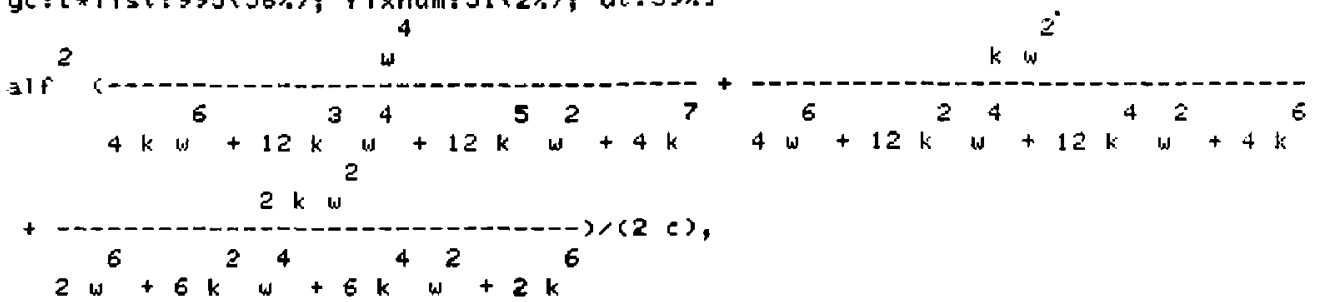

$2 w^{6}+6 k^{2} w^{4}+6 k^{4} w^{2}+2 k^{6}$

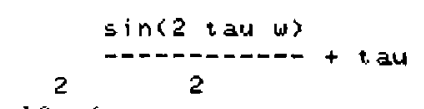

$2\left\langle-\frac{\sin \langle\operatorname{tau} w\rangle}{2}-\frac{\operatorname{tau} w}{2} \frac{2}{2}\right.$

$\exists 1$

$2 u^{3}$

w

$w+k+2 \sin (t a u w\rangle$

2

(W)

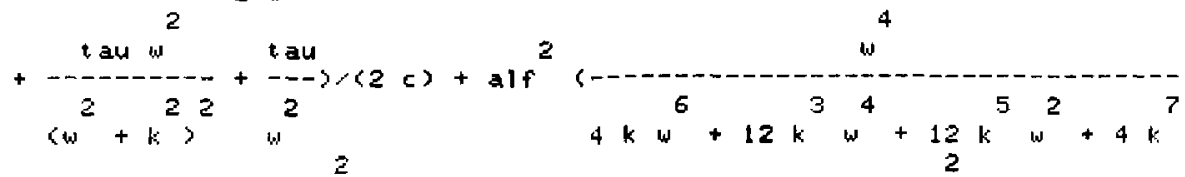

$k w^{2} \quad 2 k w$

6
6

$4 w+12 k^{2} w^{4}+12 k^{4} w^{2}+4 k^{6} 2 w^{6}+6 k^{4} w^{4}+6 k^{4} w^{2}+2 k$

alf

$4 k w^{2}+12 k^{3} 2^{4}+12 k^{5} w^{2}+4 k^{2} 4 w^{6}+12 k^{4}+12 k+w^{2}+4 k$

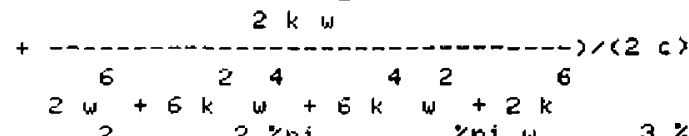

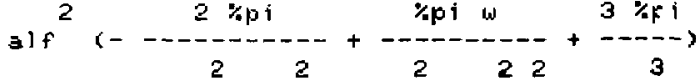

w) $(w+k)(w+k) 2 w$

+

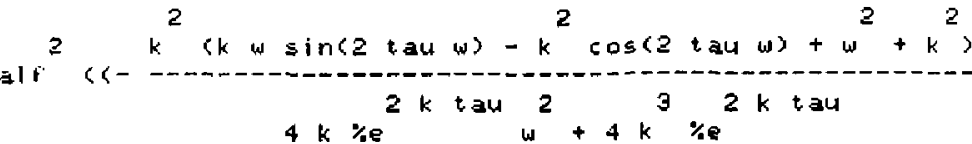

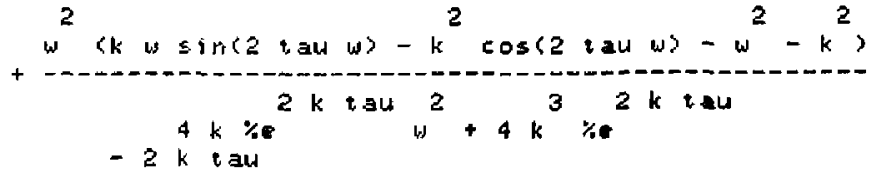




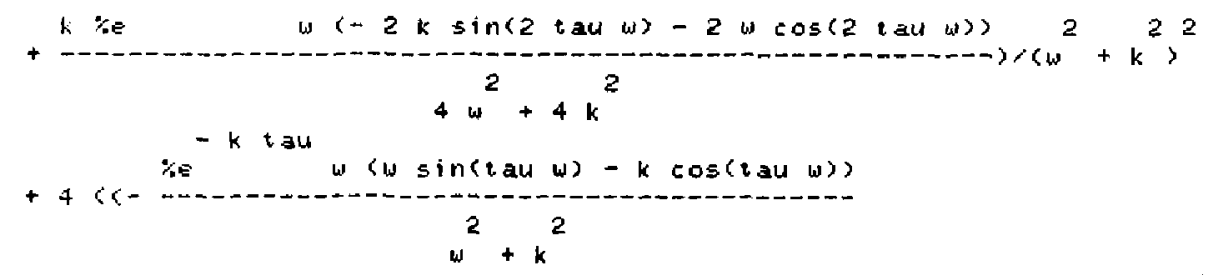




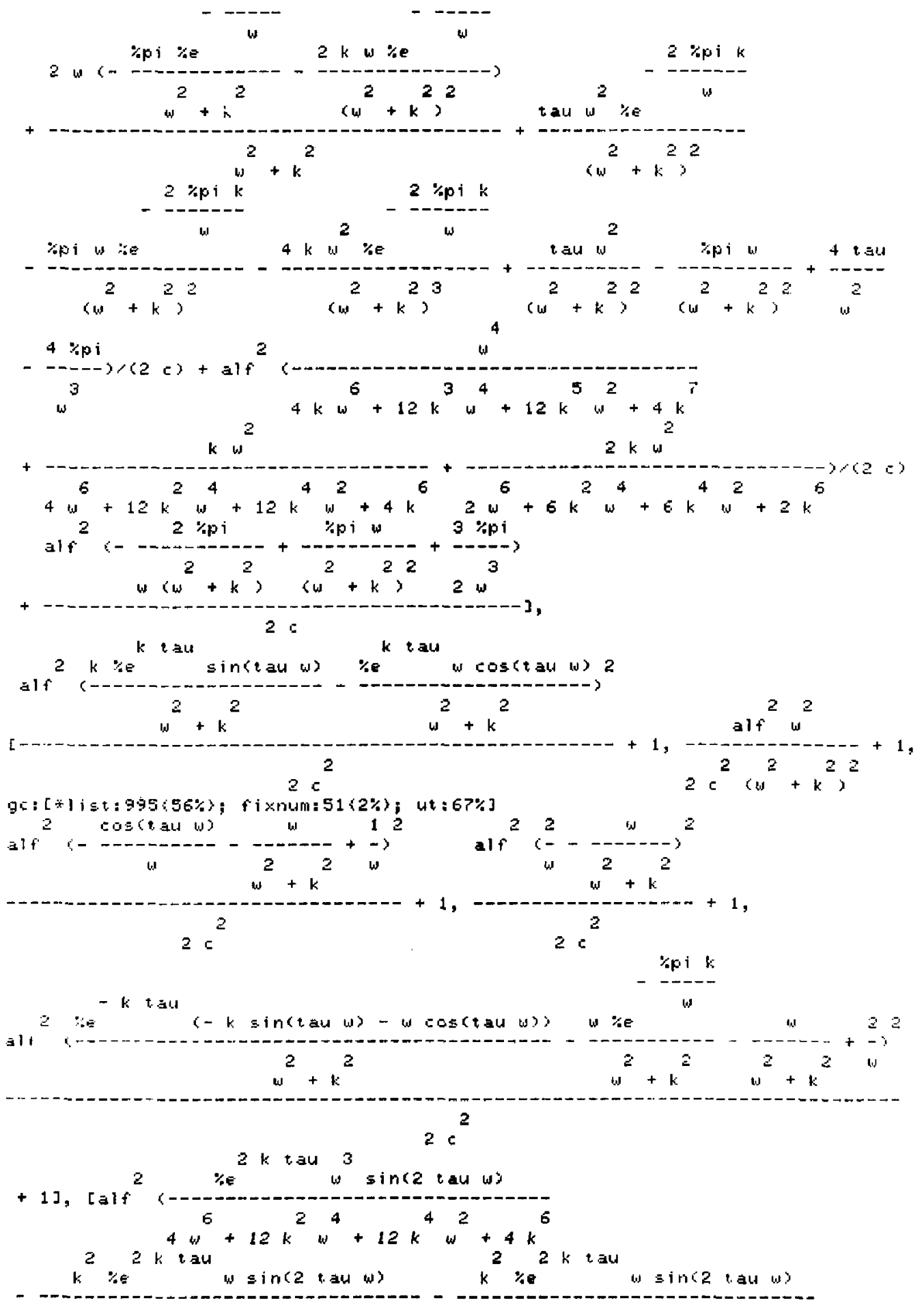




$$
\begin{aligned}
& 4 w^{6}+12 k^{2} w^{4}+12 k^{4} w^{2}+4 k^{6} 2 w^{6}+6 k^{2} w^{4}+6 k^{4} w^{2}+2 k^{6}
\end{aligned}
$$

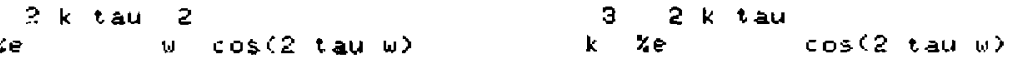

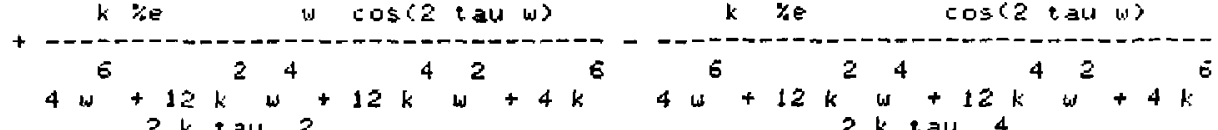

$$
\begin{aligned}
& k \pi e^{2} \cos (2 \text { tau } w) \text { ie }
\end{aligned}
$$

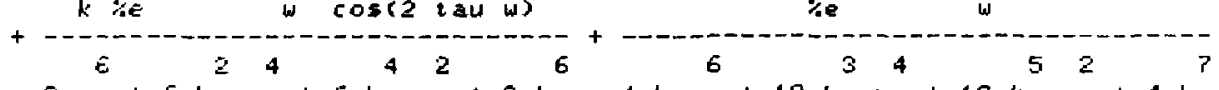

$$
\begin{aligned}
& 2 w+6 k w+6 k w+2 k+4 k w+12 k+1+12 k w+4 k \\
& 32 k \text { tul } 2 k t a u 2
\end{aligned}
$$

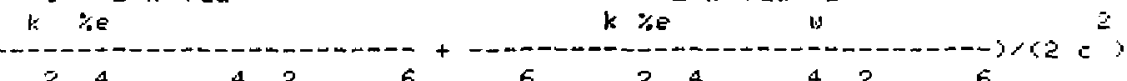

$$
\begin{aligned}
& 4 w^{6}+12 k^{2} w^{4}+12 k^{4} w^{2}+4 k^{6} 2 w^{6}+6 k^{2} w^{4}+6 k^{4} w^{2}+2 k
\end{aligned}
$$

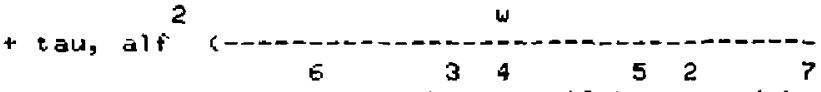

$$
\begin{aligned}
& 4 k w_{2}^{6}+12 k^{3} w^{4}+12 k^{5} w^{2}+4 k^{7} \\
& + \\
& 4 w^{2}+12 k^{2} w^{4}+12 k^{4} w^{2}+4 k^{5} \\
& 2 w^{6}+6 k^{2} w^{2}+6 k^{2} w^{2}+2 k^{6}+2
\end{aligned}
$$

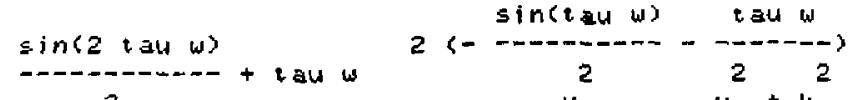

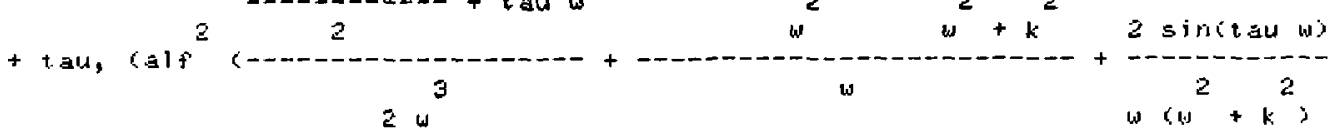

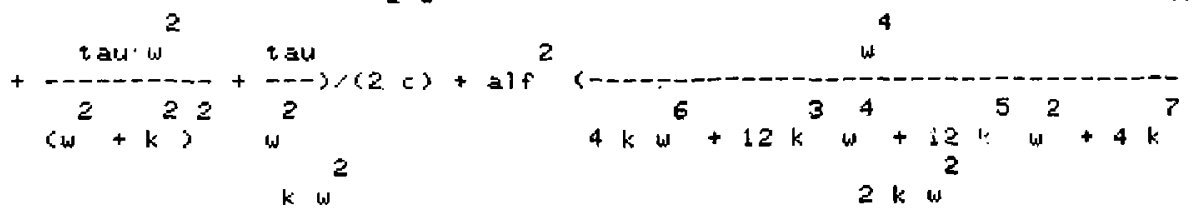

$$
\begin{aligned}
& 4 w^{6}+12 k^{2} w^{4}+12 k^{4} w^{2}+4 k^{6} 2 w^{6}+6 k^{2} w^{4}+6 k^{4} w^{2}+2 k^{6}
\end{aligned}
$$

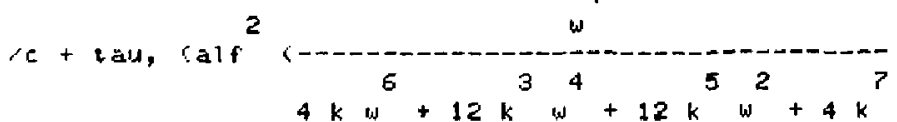

$$
\begin{aligned}
& \begin{array}{r}
4 k w+12 k w+12 k w+2 k w^{2} \\
k w
\end{array}
\end{aligned}
$$

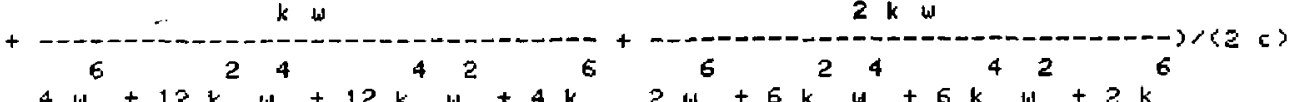

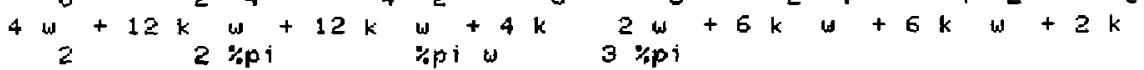

$$
\begin{aligned}
& \text { aif (- } \\
& \left.\left.w(u)^{2}+k^{2}\right)(w)^{2}+k^{2}\right)^{2} w^{3} \\
& 2 \mathrm{c} \\
& g c:[* 1 \text { ist: } 1085(64 \%) ; \text { fixnuth: } 51\{2 \%) \text {; wi:68\%] }
\end{aligned}
$$

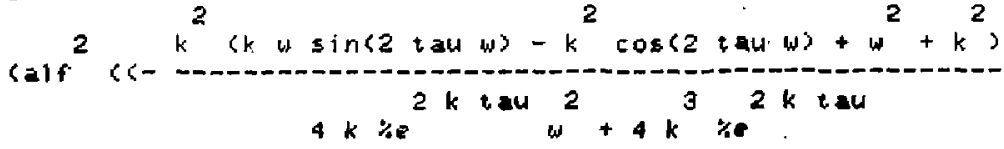




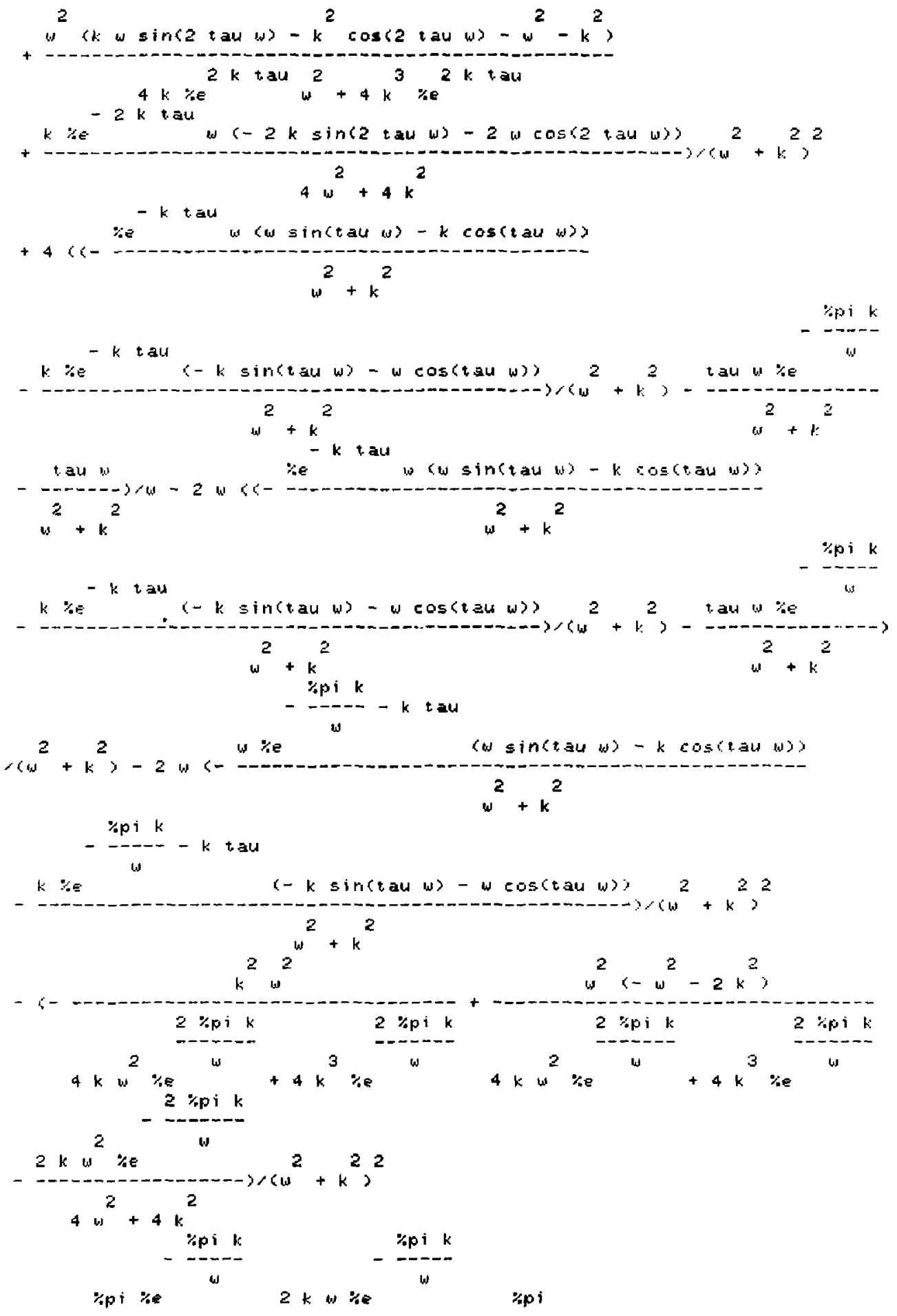




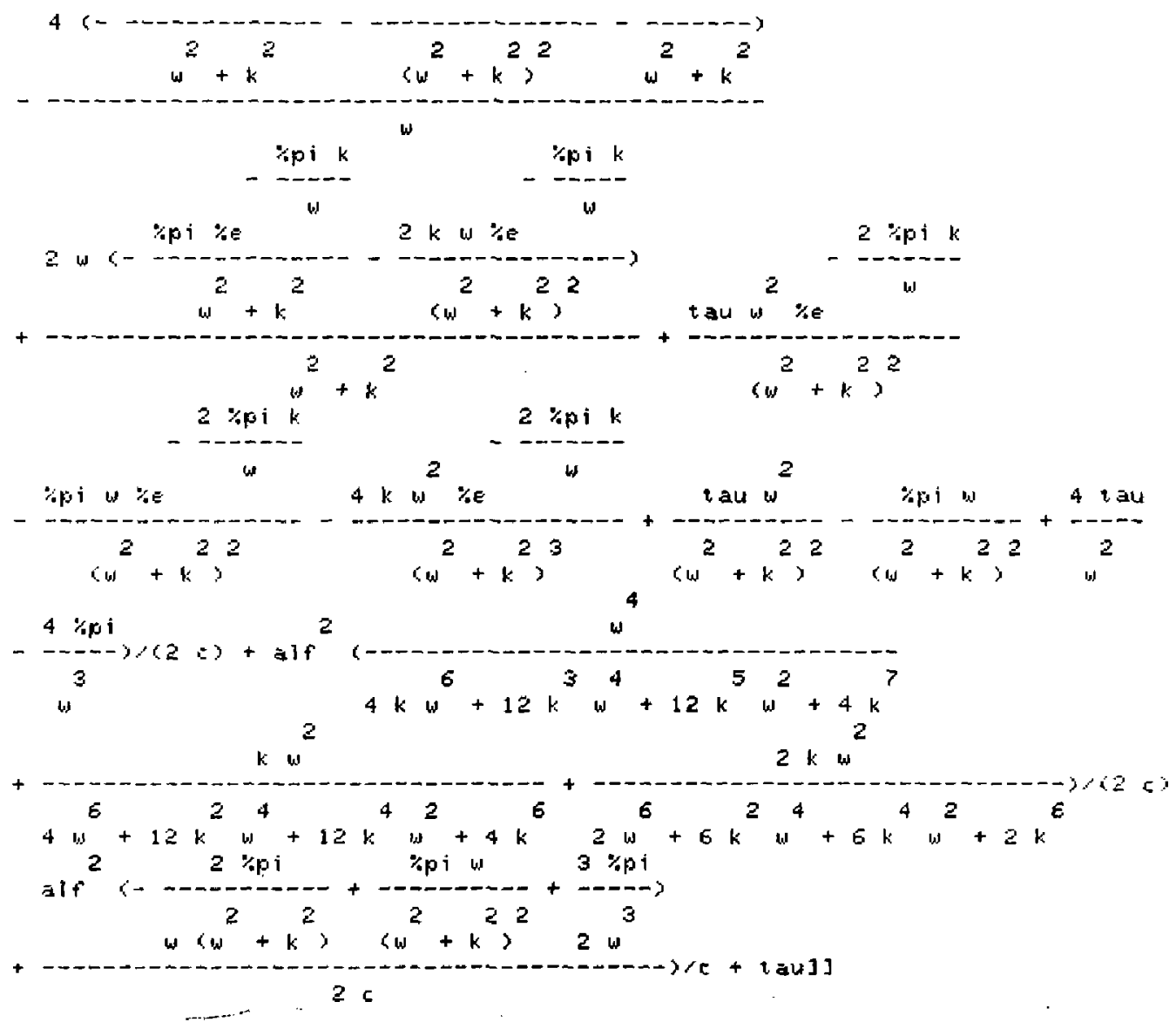

